\title{
Review: Tissue Engineering of Small-Diameter Vascular Grafts and Their In Vivo Evaluation in Large Animals and Humans
}

\author{
Shu Fang 1,2,3,*(D), Ditte Gry Ellman ${ }^{1,3}$ and Ditte Caroline Andersen $1,2,3$ (D) \\ 1 Laboratory of Molecular and Cellular Cardiology, Department of Clinical Biochemistry and Pharmacology, \\ Odense University Hospital, J. B. Winsløwsvej 25, 5000 Odense C, Denmark; dellman@health.sdu.dk (D.G.E.); \\ DAndersen@health.sdu.dk (D.C.A.) \\ 2 The Danish Regenerative Center, Odense University Hospital, J. B. Winsløwsvej 4, 5000 Odense C, Denmark \\ 3 Institute of Clinical Research, University of Southern Denmark, J. B. Winsløwsvej 19, \\ 5000 Odense C, Denmark \\ * Correspondence: sfang@health.sdu.dk or fangshu@zju.edu.cn
}

Citation: Fang, S.; Ellman, D.G.; Andersen, D.C. Review: Tissue Engineering of Small-Diameter Vascular Grafts and Their In Vivo Evaluation in Large Animals and Humans. Cells 2021, 10, 713. https:// doi.org/10.3390/cells10030713

Academic Editor: Jeremy M. Crook

Received: 10 February 2021

Accepted: 15 March 2021

Published: 23 March 2021

Publisher's Note: MDPI stays neutral with regard to jurisdictional claims in published maps and institutional affiliations.

Copyright: () 2021 by the authors. Licensee MDPI, Basel, Switzerland. This article is an open access article distributed under the terms and conditions of the Creative Commons Attribution (CC BY) license (https:// creativecommons.org/licenses/by/ $4.0 /)$.

\begin{abstract}
To date, a wide range of materials, from synthetic to natural or a mixture of these, has been explored, modified, and examined as small-diameter tissue-engineered vascular grafts (SD-TEVGs) for tissue regeneration either in vitro or in vivo. However, very limited success has been achieved due to mechanical failure, thrombogenicity or intimal hyperplasia, and improvements of the SD-TEVG design are thus required. Here, in vivo studies investigating novel and relative long (10 times of the inner diameter) SD-TEVGs in large animal models and humans are identified and discussed, with emphasis on graft outcome based on model- and graft-related conditions. Only a few types of synthetic polymer-based SD-TEVGs have been evaluated in large-animal models and reflect limited success. However, some polymers, such as polycaprolactone (PCL), show favorable biocompatibility and potential to be further modified and improved in the form of hybrid grafts. Natural polymerand cell-secreted extracellular matrix (ECM)-based SD-TEVGs tested in large animals still fail due to a weak strength or thrombogenicity. Similarly, native ECM-based SD-TEVGs and in-vitro-developed hybrid SD-TEVGs that contain xenogeneic molecules or matrix seem related to a harmful graft outcome. In contrast, allogeneic native ECM-based SD-TEVGs, in-vitro-developed hybrid SD-TEVGs with allogeneic banked human cells or isolated autologous stem cells, and in-body tissue architecture (IBTA)-based SD-TEVGs seem to be promising for the future, since they are suitable in dimension, mechanical strength, biocompatibility, and availability.
\end{abstract}

Keywords: small-diameter tissue engineered vascular grafts (SD-TEVGs); large-animal models; patency; end-to-side anastomosis; end-to-end anastomosis; antithrombotic therapy

\section{Introduction}

The leading cause of death worldwide is cardiovascular disease [1]. In the European Union countries, 119 deaths per 100,000 inhabitants in 2016 were caused by ischemic heart diseases [2]. The latter is most often caused by atherosclerosis, which also results in peripheral artery disease. The involved artery is narrowed in lumen, and the flow rate is limited, resulting in reduced blood perfusion, and oxygen and nutrients supply. Due to the development of improved medication and percutaneous intervention, surgical intervention has decreased in some areas of the world; however, bypass grafting still plays an important role for severely affected patients to recover blood perfusion.

For coronary-artery bypass grafting (CABG), the most optimal graft is autologous left internal mammary artery [3], which offers adequate diameter and length for coronaryartery revascularization [4], with a satisfying long-term patency rate of more than $85 \%$ after 10 years [5] (Table 1$)$.

The main failure reason, in the late phase, for left internal mammary artery graft is competitive flow from residual blood flow from the native coronary artery [6]. In contrast, 
the suboptimal, but most commonly used graft, is saphenous vein that displays a relatively low long-term patency rate of $61 \%$ after 10 years [6]. It often fails due to thrombosis in the early phase (within 1 month), whereas intimal hyperplasia and atherosclerosis are the failure reasons in intermediate (within 12 months) and late phases (after 12 months) [7]. Other autologous arteries (e.g., radial artery and right gastroepiploic artery) may be used alternatively for CABG; however, no prosthetic graft is approved for CABG yet [4].

For bypass grafting in lower extremity, infrainguinal bypass above the knee (femoropopliteal bypass) is considered to be a medium-diameter surgery, while infrainguinal bypass below the knee (femorodistal bypass) is considered to be a small-diameter bypass surgery (Table 1). Although the autologous saphenous vein displays a diameter usually smaller than $6 \mathrm{~mm}$, it still remains the most optimal graft for both above- and below-knee bypass surgery due to the unavailability of autologous arterial graft in general [8], but it should be noted that the primary patency rate is $53.7 \%$ after 3 years [9]. Mechanisms of saphenous vein graft failure in infrainguinal bypass are suggested to be similar to those in CABG [10]. However, unlike CABG, other non-autologous grafts (e.g., prosthetic grafts and human umbilical veins) are available for lower extremity bypass grafting above the knee with relative lower, but still comparable, primary patency rates [8]. Small-diameter bypass grafting is also performed in upper extremity but with much less incidence than bypass grafting in the heart and the lower extremities [11].

In general, arterial bypass grafting in the heart or below the knee requires smalldiameter grafts. Thus, shortage of material for such surgeries remains a big challenge because autologous grafts are often not available in certain patient groups such as claudicants, patients with diabetics or vein disease, and in patients requiring reoperations. This has further underscored the need for developing alternative small-diameter vascular grafts $[12,13]$. One candidate, small-diameter tissue-engineered vascular grafts (SD-TEVGs), is fabricated using novel techniques and interdisciplinary knowledge including material, engineering, and cell biology. Advantages of using SD-TEVGs as compared to autografts, include noninvasive surgery during preparation of grafts, unlimited availability, and customized dimension.

Table 1. Medium- and small-diameter arterial bypass grafting in clinical practice.

\begin{tabular}{|c|c|c|c|c|c|c|c|c|c|}
\hline Diseases & Bypass Site & $\begin{array}{c}\text { Host Artery } \\
\text { Diameter (mm) }\end{array}$ & $\underset{\text { Graft }}{\text { Optimal }}$ & $\begin{array}{c}\text { Graft } \\
\text { Length } \\
\text { (cm) }\end{array}$ & $\begin{array}{c}\text { Graft Diameter } \\
(\mathrm{mm})\end{array}$ & $\begin{array}{c}\text { Anastomotic } \\
\text { Configuration } \\
\text { (Distal) }\end{array}$ & $\begin{array}{l}\text { 1-Year } \\
\text { Patency }\end{array}$ & $\begin{array}{c}\text { 3-Year } \\
\text { Patency }\end{array}$ & $\begin{array}{l}\text { 10-Year } \\
\text { Patency }\end{array}$ \\
\hline $\begin{array}{l}\text { Coronary-artery } \\
\text { disease (CAD) }\end{array}$ & $\begin{array}{l}\text { Coronary- } \\
\text { artery } \\
\text { bypass }\end{array}$ & $\begin{array}{c}\text { P: } 1.6-7.2 \\
\text { M: } 1.0-6.7 \\
\text { D: } 0.8-2.5^{*}[4]\end{array}$ & $\begin{array}{c}\text { Left internal } \\
\text { mammary } \\
\text { artery [3] }\end{array}$ & $\begin{array}{c}14.3-19.5 \\
{[4]}\end{array}$ & $1.5-1.8[4]$ & End-to-side & $95 \%[5]$ & $93 \%$ [5] & $85 \%$ [5] \\
\hline $\begin{array}{c}\text { Peripheral } \\
\text { arterial disease } \\
\text { (PAD) }\end{array}$ & $\begin{array}{l}\text { Infrainguinal } \\
\text { bypass }\end{array}$ & $\begin{array}{c}\text { Femoral: } \\
\text { P: } 10.2 \\
\text { D: } 7.7 \\
\text { Popliteal: } 6.9 \\
\text { Tibial: } 3.8 / 4.2 \# \\
\text { [14] }\end{array}$ & $\begin{array}{c}\text { Great } \\
\text { saphenous } \\
\text { vein [15] }\end{array}$ & $\begin{array}{c}72.4 \pm 6.6 \\
{[16]}\end{array}$ & $\begin{array}{c}\text { P: } 5.2 \pm 0.6 \\
\text { M: } 3.3 \pm 0.5 \\
\text { D: } 1.7 \pm 0.3[16]\end{array}$ & End-to-side & $74.4 \%$ [9] & $53.7 \%$ [9] & \\
\hline
\end{tabular}

* P: proximal segment; M: media segment; D: distal segment; and \# Tibial: anterior/posterior.

\section{SD-TEVGs Evaluated in Humans}

In past decades, different types of SD-TEVGs have indeed been explored and evaluated in humans, either as arterial bypass grafts or arteriovenous shunts. To exemplify current progress, representative SD-TEVGs tested in humans are summarized in Table 2 and below.

There are several case reports and clinical trials that investigated the usage of synthetic SD-TEVGs at the aortocoronary site.

In 1976, Sauvage et al. reported about a knitted Dacron filamentous vascular prosthesis (3.5 $\mathrm{mm}$ in diameter and $4 \mathrm{~cm}$ long) as an interposition graft at the aortocoronary site in a 65-year-old patient to repair the coronary artery after removal of a saccular aneurysm in the ascending aorta [17]. This graft maintained patency during the 16-month follow-up period. Success has also been observed in similar synthetic grafts at the aortocoronary site [18]. However, considering the bypass location between the aorta and the proximal 
end of the coronary artery with high flow, these case reports did not support implantation of synthetic graft for common CABG, where the grafts need to be implanted to coronary arteries at more distal positions [17].

From 1982 to 2008, at least six types of grafts were further evaluated in patients that underwent CABG [19] (Table 2):

(1) glutaraldehyde-fixed human umbilical vein grafts with a patency of $46 \%$ after 3-13-month follow-up published in 1982 [20];

(2) cryopreserved allograft saphenous vein with a patency of $41 \%$ after $2-16$-month follow-up published in 1992 [21];

(3) dialdehyde starch-treated bovine internal thoracic artery grafts with a patency of 16\% after 3-23-month follow-up in 1993 [22];

(4) No-React bovine internal mammary artery with a patency of 57\% after 1-4.5-year follow-up in a study in 2004 [23] and a patency of 23\% after 3-11-month follow-up in another study in 2008 [24];

(5) autologous endothelial cell-seeded expanded polytetrafluoroethylene (ePTFE) grafts with a patency of $90.5 \%$ after 7.5-48-month follow-up in 2000 [25];

(6) de-endothelialized and cryopreserved allograft veins seeded by autologous endothelial cells with a patency of 50\% after 9-month follow-up published in 2001 [26] and $0 \%$ patency after 32 months, published in 2019 [27].

The first four types of grafts showed very poor patency and therefore were not recommended as alternative choices for CABG in patients, whereas the fifth type of graft displayed high patency [25], suggesting promising improvement of graft patency by endothelialization as also discussed below. This improvement of endothelialization was also seen in the allograft veins seeded by autologous endothelial cell [26,27], as compared to the similar cryopreserved allograft saphenous vein but without endothelialization [21]. However, when comparing the two types of grafts that were both endothelialized [25-27], the synthetic ePTFE [25] seems much better than the cryopreserved allograft [26,27], indicating that elimination of immunogenicity in the allografts cannot be fully achieved by cryopreservation and therefore need to be further improved by using other methodology such as decellularization. Thus, until now, modified synthetic, allogeneic, or xenogeneic grafts have indeed been studied in humans for CABG, however with very limited success due to their thrombogenicity. Furthermore, there are no human studies testing SD-TEVGs for CABG initiated after 2008.

In regard to human studies for artery bypass grafting below the knee, Almasri et al. reviewed large-scale clinical trials in 2018 and revealed a primary patency around 50\% of FDA-approved prosthetic grafts (cryopreserved saphenous vein allografts and heparin bounded polytetrafluoroethylene (PTFE)) at 1-year follow-up using meta-analysis [28]. Recently, another type of FDA-approved TEVG termed crosslinked bovine carotid artery graft (BCAG) has been examined in patients for artery bypass grafting below the knee. They display a long-term primary patency at 50-75\% 5 years after implantation (Table 2) [29], which is comparable to autologous vein graft and might be better than synthetic grafts [28]. However, the study was retrospective, and therefore, prospective randomized studies are needed to compare these xenogeneic grafts with autologous vein grafts and synthetic grafts. To reduce the thrombogenicity of synthetic grafts, Williams et al. recellularized ePTFE with autologous adipose-derived stromal vascular fraction cells and implanted these modified grafts as femoral-to-tibial bypass grafts in a phase 1 clinical trial (Table 2) [30]. The 1 year patency of these recellularized grafts was $60 \%$ (3/5 were patent). 
Table 2. Small-diameter tissue-engineered vascular grafts (SD-TEVGs) evaluated in humans.

\begin{tabular}{|c|c|c|c|c|c|c|c|}
\hline Author & Graft Type & Year & Graft & Number of Patients & Recellularization & Follow-Up Time & Primary Patency \\
\hline \multicolumn{8}{|c|}{ CABG } \\
\hline Silver [20] & Allogeneic & 1982 & $\begin{array}{c}\text { Glutaraldehyde-fixed human umbilical } \\
\text { vein grafts }\end{array}$ & 11 & None & 3 to 13 months & $46 \%$ \\
\hline Laub [21] & Allogeneic & 1992 & Cryopreserved allograft saphenous vein & 19 & None & 2 to 16 months & $41 \%$ \\
\hline Mitchell [22] & Xenogeneic & 1993 & $\begin{array}{l}\text { Dialdehyde starch-treated bovine internal } \\
\text { mammary artery }\end{array}$ & 18 & None & 3 to 23 months & $16 \%$ \\
\hline Reddy [23] & Xenogeneic & 2004 & No-React bovine internal mammary artery & 7 & None & 1 to 4.5 years & $57 \%$ \\
\hline Englberger [24] & Xenogeneic & 2008 & No-React bovine internal mammary artery & 17 & None & 3 to 11 months & $23 \%$ \\
\hline $\begin{array}{l}\text { Lamm [26] and } \\
\text { Herrmann [27] }\end{array}$ & $\begin{array}{l}\text { Autologous } \\
\text { cells on } \\
\text { allograft }\end{array}$ & $\begin{array}{l}2001 \text { and } \\
2019\end{array}$ & $\begin{array}{c}\text { Deendothelialized/cryopreserved allograft } \\
\text { veins seeded by autologous endothelial } \\
\text { cells }\end{array}$ & 12 & $\begin{array}{l}\text { Autologous } \\
\text { endothelial cell }\end{array}$ & 16 to 18 years & $\begin{array}{l}80 \% \text { ( } 6 \text { months); } \\
50 \% \text { (9 months); } \\
0 \% \text { (32 months) }\end{array}$ \\
\hline \multicolumn{8}{|c|}{ Bypass grafting below knee } \\
\hline Lindsey [29] & Xenogeneic & 2017 & Crosslinked bovine carotid artery & 80 & None & 5 years & $52 \%$ to $75 \%$ \\
\hline Williams [30] & $\begin{array}{l}\text { Autologous } \\
\text { cells on } \\
\text { synthetic }\end{array}$ & 2017 & $\begin{array}{l}\text { Adipose-Derived Stromal Vascular Fraction } \\
\text { Cell seeded ePTFE }\end{array}$ & 5 & $\begin{array}{l}\text { Adipose-Derived } \\
\text { Stromal Vascular } \\
\text { Fraction Cell }\end{array}$ & 1 year & $60 \%$ \\
\hline \multicolumn{8}{|c|}{ AV shunt for hemodialysis access } \\
\hline Kennealey [31] & Xenogeneic & 2011 & Crosslinked bovine carotid artery & 26 & None & 1 year & $61 \%$ \\
\hline Harlander-Locke [32] & Xenogeneic & 2014 & Crosslinked bovine carotid artery & 17 & None & 18 months & $73 \%$ \\
\hline Wystrychowski [33] & Allogeneic & 2014 & $\begin{array}{c}\text { Allogeneic cell sheet-based TEVG, } \\
\text { dehydrated }\end{array}$ & 3 & None & $<11$ months & $\begin{array}{l}9.5 \text { patient-month } \\
\text { of use }\end{array}$ \\
\hline Lawson [34] & Allogeneic & 2016 & Allogeneic human acellular vessels & 60 & $\begin{array}{c}\text { None } \\
\text { Autologous }\end{array}$ & $>1$ year & $\begin{array}{c}28 \% \text { at } 12 \text { months } \\
24\end{array}$ \\
\hline L'Heureux [35] & Autologous & 2007 & Autologous cell sheet-based TEVG & 6 & $\begin{array}{l}\text { fibroblast and } \\
\text { endothelial cells }\end{array}$ & $<13$ months & $\begin{array}{l}\text { patient-months of } \\
\text { use }\end{array}$ \\
\hline McAllister [36] & Autologous & 2009 & Autologous cell sheet-based TEVG & 10 & $\begin{array}{l}\text { Autologous } \\
\text { fibroblast and } \\
\text { endothelial cells }\end{array}$ & $>6$ months & $\begin{array}{c}68 \\
\text { patient-months of } \\
\text { use }\end{array}$ \\
\hline Wystrychowski [37] & Autologous & 2011 & $\begin{array}{l}\text { Autologous cell sheet-based TEVG, } \\
\text { cold-preserved }\end{array}$ & 1 & $\begin{array}{l}\text { Autologous } \\
\text { endothelial cells }\end{array}$ & 8 weeks & $\begin{array}{l}8 \text { patient-weeks } \\
\text { of use }\end{array}$ \\
\hline
\end{tabular}


Instead of CABG and bypass grafting in lower extremity, arteriovenous shunt for hemodialysis in patients with end-stage renal disease has become a popular model for testing novel TEVGs (Table 2), since adverse events like graft failure are less likely to harm these patients. Since 2007, L'Heureux and colleagues have focused on testing novel cell-sheet-based TEVGs in patients as hemodialysis access. As such, they have assessed autologous fully recellularized TEVGs $[35,36]$, autologous cold-preserved TEVGs with endothelial cells [37], and allogeneic dehydrated TEVGs [33]. However, in all three types of grafts either poor mechanical properties or poor patency outcome were apparent, as dilation, aneurysm, and thrombus were often observed. In contrast, the TEVGs developed by Lawson and colleagues, using cell-sheet-based technology and decellularization, manifested stable mechanical strength over time. Although a poor primary patency was observed in this study, the secondary patency of the cell-sheet-based TEVGs was found fairly positive at $89 \%$ after more than 1 year follow-up. Moreover, as compared to the synthetic grafts tested, the cell-sheet-based TEVGs possessed higher resistance to prosthetic infection, which is a common reason for graft failure in arteriovenous shunt for hemodialysis access [34]. Crosslinked BCAG has also been suggested as an alternative to autologous grafts. When implanted in patients as arteriovenous shunt for hemodialysis access, crosslinked BCAG exhibit a patency of $60 \%$ to $70 \%$ after 12 or 18 months [31,32], which is similar to the positive outcome observed in lower extremity bypass grafting [29].

Thus, although some progress has been achieved regarding SD-TEVGs in clinical studies, autologous arteries or veins are still superior and the first choice for small-diameter artery bypass grafting. However, techniques in this field develop at a high speed (see below), and progress is substantiated by the large number of studies testing SD-TEVGs in large animals.

\section{SD-TEVG Studies in Large-Animal Models}

\subsection{Systematic Search}

Large-animal models are regarded as important preclinical tools to determine the quality and functionality of novel SD-TEVGs. They are more similar to humans with regards to the coagulation system, hemodynamics, and hematological profiles as compared to small animals [38,39], although rodent and rabbit models are widely used to test SDTEVGs as an alternate to human and large-animal models [40,41]. To further provide a status overview on the field of SD-TEVGs, we therefore used a systematic approach to identify studies that investigate SD-TEVGs as a relative long piece (longer than 10 times the inner diameter) in large-animal models. Accordingly, studies from 1 January 1995, until 1 November 2019, were found on PubMed by searching: "vascular graft", "tissue engineering", "smameter", and "in vivo" according to inclusion and exclusion criteria that apply to a strategy of clinical translation (Table 3).

Table 3. Literature review strategies for SD-TEVGs in large animals.

\begin{tabular}{|c|c|c|}
\hline & Inclusion Criteria & Exclusion Criteria \\
\hline 1 & In vivo in large animal (>rabbit) & In vitro or in small animal ( $\leq$ rabbit) or in human \\
\hline 2 & Inner diameter $\leq 6 \mathrm{~mm}$ & Inner diameter $>6 \mathrm{~mm}$ \\
\hline 3 & $\begin{array}{l}\text { Bypass at small-/medium-diameter artery site } \\
\text { (E.g.: coronary, carotid or femoral artery) }\end{array}$ & $\begin{array}{l}\text { Bypass at large-diameter artery site or venous system } \\
\text { (E.g.: aorta, aortoiliac artery bypass pulmonary vein or artery, } \\
\text { cavopulmonary connection, and venous bypass) }\end{array}$ \\
\hline 4 & $\begin{array}{l}\text { Graft evaluated as arterial bypass graft or } \\
\text { arteriovenous shunt }\end{array}$ & $\begin{array}{l}\text { Graft evaluated as microvascular network, microvessels, stent, } \\
\text { valve, or patch }\end{array}$ \\
\hline 5 & Graft length $\geq 10$ times of diameter & Graft length $<10$ times of diameter \\
\hline
\end{tabular}

Specifically, earlier studies have suggested that a limited length $(<10$ times the inner diameter) might overevaluate the patency of grafts [42,43]. In addition, considering the clinical need for substitutes of relative long $(>20 \mathrm{~cm})$ TEVGs for bypass grafting, we only included grafts with a length more than 10 times the diameter, which are believed to be 
much more clinically relevant regarding to bypass grafting in humans [42]. Furthermore, considering the high flow rate at the aorta site and low pressure in the venous and pulmonary system, only studies concerning arterial small-diameter engraftment sites like the coronary-artery bed and medium-diameter sites like the carotid or femoral vasculature were included (Table 3). In this regard, it is important to note that SD-TEVGs are required for femerodistal or femeropopliteal bypasses, even though the femoral artery possesses a diameter larger than $7 \mathrm{~mm}$ (Table 1).

A total of 39 studies were included (Tables 4-7), and information regarding modelrelated conditions (animal, bypass site, anastomosis fashion, and usage of antithrombotic treatment), graft-related parameters (material, origin, modification, recellularization, and mechanical modification) and outcome (follow-up, patency, and failure mechanism) were extracted and stratified (Tables 4-7). Notably, some studies represented more than one type of TEVG or more than one insertion site, etc., and data are thus categorized separately in our stratifications.

Regarding insertion site, 32 studies used arterial bypass (at coronary-artery sites (1 study), in carotid (27 studies), as femoral arteries (six studies) (Tables 4-6)), whereas nine studies concerned arteriovenous shunts (Table 7). Furthermore, in studies using arterial bypass, six studies used an end-to-side (ETS) anastomotic surgery technique while 25 performed end-to-end (ETE), and one study did not specify regarding this (Tables 4-6). In contrast, ETS was most often performed in arteriovenous shunt due to its anatomical nature (Table 7). Overall, 26 studies used systemic antithrombotic treatment, while nine studies did not use such treatment, and two studies included evaluation of grafts under both conditions for direct comparisons [44,45], while two studies did not reveal if systemic antithrombotic treatment was applied [46,47] (Tables 4-7). Using these stratifications (Tables 4-7), we discuss below the different types of SD-TEVGs and their potential for future clinical translation.

\subsection{Tissue Engineering of Small-Diameter Vascular Grafts}

In general, tissue engineering is referred to as an interdisciplinary field that combines biological and engineering knowledge as well as their techniques to develop viable tissue or organs for patients [48]. To date, SD-TEVGs composed of a diverse array of materials ranging from synthetic to natural or a mixture hereof have been explored and eventually modified to examine their functionality as porous scaffold for supporting cell growth and tissue regeneration either in vitro or in vivo (Figure 1 and Table 8).
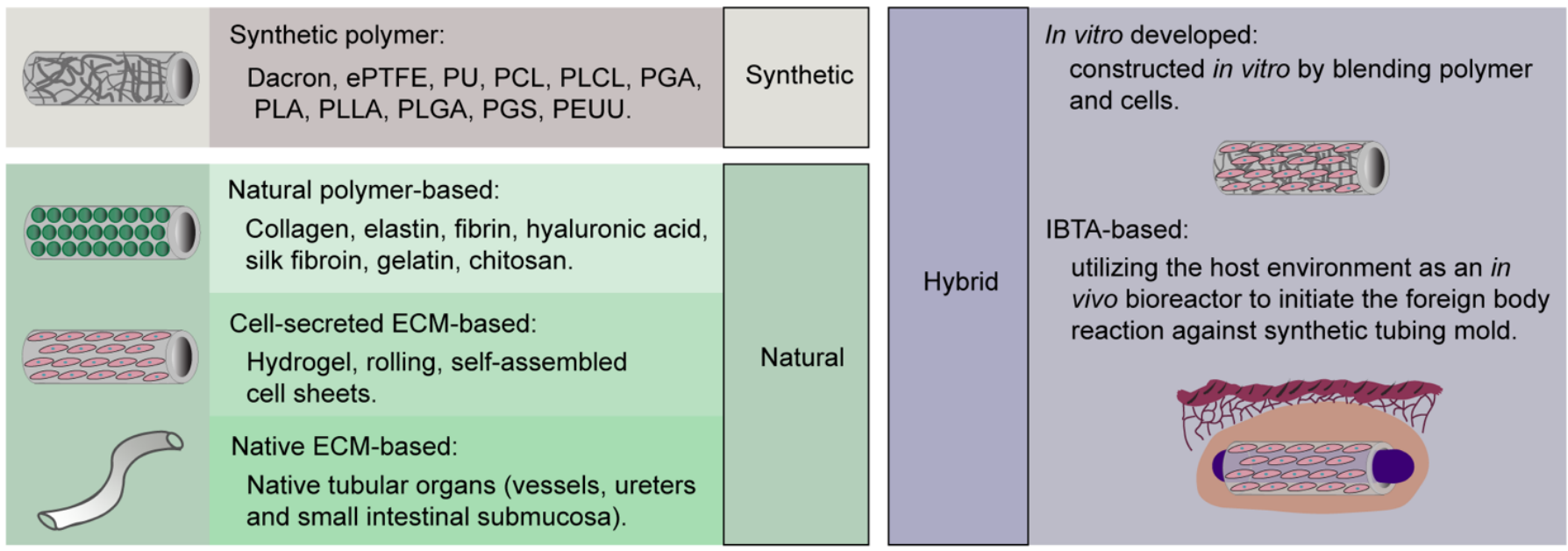

Figure 1. Classification of SD-TEVGs tested in large animals as arterial bypass graft or arteriovenous shunt with length $\geq$ 10 times the diameter. IBTA: in-body tissue architecture. 
Table 4. SD-TEVGs evaluated in large animals in arterial bypass using end-to-side (ETS) anastomosis.

\begin{tabular}{|c|c|c|c|c|c|c|c|c|c|c|c|c|c|c|c|c|}
\hline \multirow[t]{2}{*}{ Study Group } & \multicolumn{6}{|c|}{ Model } & \multicolumn{2}{|c|}{ Graft } & \multicolumn{5}{|c|}{ Modification } & \multicolumn{3}{|c|}{ Outcome } \\
\hline & $\underset{(\mathrm{mm})}{\mathrm{D}}$ & $\underset{(\mathrm{cm})}{\mathrm{L}}$ & Animal & Implantation site & Anastomosis & $\begin{array}{l}\text { Antithro- } \\
\text { mbotic } \\
\text { therapy }\end{array}$ & Graft type & Material & $\begin{array}{l}\text { Chemical } \\
\text { Modification }\end{array}$ & $\begin{array}{c}\text { Biological } \\
\text { modification = } \\
\text { Recellulariza- } \\
\text { tion }\end{array}$ & $\begin{array}{l}\text { Luminal } \\
\text { cell type }\end{array}$ & $\begin{array}{c}\text { Medial cell } \\
\text { type }\end{array}$ & $\begin{array}{l}\text { Mechanical } \\
\text { modification }= \\
\text { Precondition }\end{array}$ & $\begin{array}{l}\text { Follow-up } \\
\text { (day) }\end{array}$ & Patency & Graft Failure \\
\hline $\begin{array}{l}\text { Mahara 2015 [49] } \\
7 \text { days control }\end{array}$ & 2 & 25 & Pig & $\begin{array}{l}\text { Femorale-femoral } \\
\text { artery crossover } \\
\text { bypass }\end{array}$ & $\begin{array}{l}\text { Proximal: } \\
\text { STE Distal: } \\
\text { ETE }\end{array}$ & No & Xenogeneic & $\begin{array}{l}\text { Acellular ostrich } \\
\text { carotid artery }\end{array}$ & None & None & None & None & No & 7 & 0 & Thrombus \\
\hline $\begin{array}{l}\text { Mahara 2015 [49] } \\
\text { Peptide-modified }\end{array}$ & 2 & 25 & Pig & $\begin{array}{l}\text { Femorale-femoral } \\
\text { artery crossover } \\
\text { bypass }\end{array}$ & $\begin{array}{l}\text { Proximal: } \\
\text { STE Distal: } \\
\text { ETE }\end{array}$ & No & Xenogeneic & $\begin{array}{l}\text { Acellular ostrich } \\
\text { carotid artery }\end{array}$ & POG7G3REDV & None & None & None & No & 20 & $83 \%$ & $\begin{array}{l}\text { Unstable suturing } \\
\text { at proximal } \\
\text { anastomotic site }\end{array}$ \\
\hline $\begin{array}{l}\text { Fang } 2019 \text { [HUA } \\
\text { [HU] }\end{array}$ & 4 & 4 & Sheep & Carotid artery & ETS & No & Xenogeneic & $\begin{array}{c}\text { Decellularized } \\
\text { human } \\
\text { umbilical artery }\end{array}$ & None & None & None & None & No & 28 & 0 & Thrombus \\
\hline $\begin{array}{l}\text { Fang } 2019[50] \\
\quad \text { dSCA }\end{array}$ & 4 & 4 & Sheep & Carotid artery & ETS & No & Allogeneic & $\begin{array}{l}\text { Decellularized } \\
\text { sheep carotid } \\
\text { artery }\end{array}$ & None & None & None & None & No & 28 & 0 & Distal stenosis \\
\hline $\begin{array}{l}\text { Fang [45] } \\
\text { PCL } w / o \\
\text { enoxaparin }\end{array}$ & 4 & 4 & Sheep & Carotid artery & ETS & No & Synthetic & PCL & None & None & None & None & No & 28 & 0 & Thrombus \\
\hline $\begin{array}{l}\text { Dahl } 2011 \text { [51] } \\
\text { Dog coronary, } 1 \\
\text { month }\end{array}$ & 3 or 4 & $4-8.5$ & Dog & $\begin{array}{l}\text { Coronary or } \\
\text { carotid artery }\end{array}$ & ETS * & Yes & $\begin{array}{l}\text { Autologous } \\
\text { cells on } \\
\text { allograft }\end{array}$ & $\begin{array}{l}\text { Decellularized } \\
\text { graft from } \\
\text { allogeneic } \\
\text { canine cells } \\
\text { grow on a PGA } \\
\text { scaffold }\end{array}$ & None & Autologous & Vessel-EC & None & Yes & $7-365$ & $83 \%$ & NR \\
\hline $\begin{array}{l}\text { Fang }[45] \\
\text { PCL } W \\
\text { enoxaparin }\end{array}$ & 4 & 4 & Sheep & Carotid artery & ETS & Yes & Synthetic & PCL & None & None & None & None & No & 28 & $100 \%$ & No failure \\
\hline $\begin{array}{l}\text { Soldani } 2010[53] \\
\text { ePTFE } 6 \text { months }\end{array}$ & 7 & 5 & Sheep & Carotid artery & $\begin{array}{l}\text { ETS + } \\
\text { carotid } \\
\text { resection }\end{array}$ & Yes & Synthetic & ePTFE & None & None & None & None & No & 180 & $50 \%$ & Thrombus \\
\hline $\begin{array}{l}\text { Soldani } 2010[53] \\
\text { PEtU } 24 \text { months }\end{array}$ & 7 & 5 & Sheep & Carotid artery & $\begin{array}{l}\text { Proximal: } \\
\text { ETE } \\
\text { Distal: ETS }\end{array}$ & Yes & Synthetic & $\mathrm{PU}$ & None & None & None & None & No & 730 & $100 \%$ & No failure \\
\hline
\end{tabular}

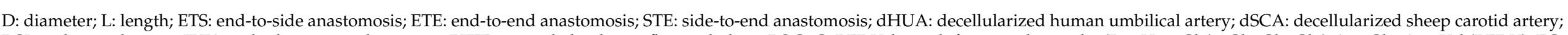

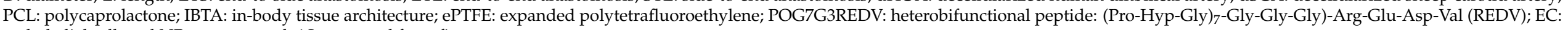
endothelial cell; and NR: not reported. * Interpreted from figure. 
Table 5. SD-TEVGs evaluated in large animal in arterial bypass using end-to-end (ETE) anastomosis (without antithrombotic therapy).

\begin{tabular}{|c|c|c|c|c|c|c|c|c|c|c|c|c|c|c|c|c|}
\hline \multirow[t]{2}{*}{ Study Group } & \multicolumn{6}{|c|}{ Model } & \multicolumn{2}{|r|}{ Graft } & \multicolumn{5}{|c|}{ Modification } & \multicolumn{3}{|c|}{ Outcome } \\
\hline & $\underset{(\mathrm{mm})}{\mathrm{D}}$ & $\underset{(\mathrm{cm})}{\mathrm{L}}$ & Animal & $\begin{array}{l}\text { Implantation } \\
\text { site }\end{array}$ & Anastomosis & $\begin{array}{l}\text { Antithro- } \\
\text { mbotic } \\
\text { therapy }\end{array}$ & Graft type & Material & $\begin{array}{l}\text { Chemical } \\
\text { Modifica- } \\
\text { tion }\end{array}$ & $\begin{array}{c}\text { Biological } \\
\text { modificition= } \\
\text { Recellularization }\end{array}$ & $\underset{\substack{\text { Luminal cell } \\
\text { type }}}{\text {. }}$ & Medial cell type & $\underset{\substack{\text { Mechanical } \\
\text { modificition }=\\
\text { Precondition }}}{\text {. }}$ & $\begin{array}{l}\text { Follow-up } \\
\text { (day) }\end{array}$ & Patency & Graft Failure \\
\hline $\begin{array}{l}\text { Aper } 2016 \text { [54] } \\
1 \text { month }\end{array}$ & 5.6 & 9 & Sheep & Carotid artery & ETE & No & $\begin{array}{l}\text { Natural (xenogeneic } \\
\text { fibrin) }\end{array}$ & $\begin{array}{l}\text { Highly compacted human } \\
\text { fibrin matrix }\end{array}$ & Factor XIII & Autologous & PB-EC & PB-SMC & No & 30 & $33 \%$ & Rupture \\
\hline $\begin{array}{l}\text { Aper } 2016 \text { [54] } \\
6 \text { months }\end{array}$ & 5.6 & 9 & Sheep & Carotid artery & ETE & No & $\begin{array}{l}\text { Natural (xenogeneic } \\
\text { fibrin) }\end{array}$ & $\begin{array}{l}\text { Highly compacted human } \\
\text { fibrin matrix }\end{array}$ & Factor XIII & Autologous & PB-EC & PB-SMC & No & 180 & $100 \%$ & No failure \\
\hline $\begin{array}{l}\text { Cho } 2005[55] \\
\text { Acellular control }\end{array}$ & 3 & 4 & Dog & Carotid artery & ETE & No & Allogeneic & $\begin{array}{l}\text { Decellularized canine carotid } \\
\text { arteries }\end{array}$ & None & None & None & None & No & 14 & 0 & Thrombus \\
\hline $\begin{array}{l}\text { Cho } 2005[55] \\
\text { BMC }\end{array}$ & 3 & 4 & Dog & Carotid artery & ETE & No & $\begin{array}{l}\text { Autologous cells on } \\
\text { allograft }\end{array}$ & $\begin{array}{l}\text { Decellularized canine carotid } \\
\text { arteries }\end{array}$ & None & Autologous & BMMNC-EC & BMMNC-SMC & No & 56 & $33 \%$ & Thrombus \\
\hline $\begin{array}{l}\text { Dahan } 2017[46] \\
\text { Acellular control }\end{array}$ & 4 & 4.5 & Pig & Carotid artery & ETE & $\begin{array}{c}\text { Not } \\
\text { mentioned }\end{array}$ & Allogeneic & $\begin{array}{l}\text { Decellularized porcine carotid } \\
\text { artery }\end{array}$ & None & None & None & None & No & 42 & $100 \%$ & $\begin{array}{l}\text { Even patent but still } \\
\text { very narrowed } \\
\text { lumen according to } \\
\text { the staining }\end{array}$ \\
\hline $\begin{array}{l}\text { Dahan 2017 [46] } \\
\text { scaECM }^{2}\end{array}$ & 4 & 4.5 & Pig & Carotid artery & ETE & $\begin{array}{c}\text { Not } \\
\text { mentioned }\end{array}$ & $\begin{array}{l}\text { Autologous cells on } \\
\text { allograft }\end{array}$ & $\begin{array}{l}\text { Decellularized porcine carotid } \\
\text { artery }\end{array}$ & None & Autologous & Vein-EC & Artery-SMC & Yes & 42 & $100 \%$ & No failure \\
\hline $\begin{array}{l}\text { He } 2002[56] \\
\text { Type A, } 1 \text { month }\end{array}$ & 5 & 5 & Dog & Carotid artery & ETE & No & $\begin{array}{l}\text { Autologous cells on } \\
\text { synthetic and natural } \\
\text { graft }\end{array}$ & $\begin{array}{l}\text { Autologoous SCSS-inouculated } \\
\text { bovine collagen gel layer and } \\
\text { an E C monloyer wrapped } \\
\text { with PU-nylon mesh }\end{array}$ & None & Autologous & Vein-EC & Vein-SMC & No & 30 & $100 \%$ & $\begin{array}{l}\text { No failure, but } \\
\text { dilation I } \\
\text { delamination was } \\
\text { seen }\end{array}$ \\
\hline $\begin{array}{l}\text { He } 2002[56] \\
\text { yype B, } 6 \text { months }\end{array}$ & 5 & 5 & Dog & Carotid artery & ETE & No & $\begin{array}{l}\text { Autologous cells on } \\
\text { synthetic and natural } \\
\text { graft }\end{array}$ & $\begin{array}{l}\text { Autologous SMCS-inoculated } \\
\text { bovine collagen gel layer and } \\
\text { an EC monolayer wrapped } \\
\text { with an excimer raser-irected } \\
\text { microporous SPU film }\end{array}$ & None & Autologous & Vein-EC & Vein-SMC & No & 180 & $100 \%$ & No failure \\
\hline $\begin{array}{c}\text { He 2003 [57] } \\
1 \text { month }\end{array}$ & 4.5 & 6 & Dog & Carotid artery & ETE & No & $\begin{array}{l}\text { Autologous cells on } \\
\text { synthetic and natural } \\
\text { graft }\end{array}$ & $\begin{array}{l}\text { Bovine collagen type I meshes } \\
\text { wrapped with a SPU thin film }\end{array}$ & None & Autologous & PB-EPCs & None & No & 30 & $83 \%$ & $\begin{array}{c}\text { Dilation and } \\
\text { thrombus }\end{array}$ \\
\hline $\begin{array}{c}\text { He 2003 [57] } \\
3 \text { months }\end{array}$ & 4.5 & 6 & Dog & Carotid artery & ETE & No & $\begin{array}{l}\text { Autologous cells on } \\
\text { synthetic and natural } \\
\text { graft }\end{array}$ & $\begin{array}{l}\text { Bovine collagen type I meshes } \\
\text { wrapped with a SPU thin film }\end{array}$ & None & Autologous & PB-EPCs & None & No & 90 & $100 \%$ & No failure \\
\hline $\begin{array}{l}\text { Narita } 2008 \text { [58] Acellular } \\
\text { DU control }\end{array}$ & 3 & 4.5 & Dog & Carotid artery & ETE & No & Allogeneic & Decellularized ureters & None & None & None & None & No & 56 & $20 \%$ & NR \\
\hline $\begin{array}{l}\text { Narita } 2008[58] \mathrm{DU}+\mathrm{EC} \\
+ \text { my fibroblasts }\end{array}$ & 3 & 4.5 & Dog & Carotid artery & ETE & No & $\begin{array}{l}\text { Autologous cells on } \\
\text { allograft }\end{array}$ & Decellularized ureters & None & Autologous & Vein-EC & Myofibroblasts & No & 168 & $100 \%$ & No failure \\
\hline $\begin{array}{l}\text { Narita } 2008[58] \\
\text { PTFE control }\end{array}$ & 3 & 4.5 & Dog & Carotid arterial & ETE & No & Synthetic & PTFE & None & None & None & None & No & 7 & 0 & NR \\
\hline $\begin{array}{l}\text { Scherner 2014 [59] } \\
\text { BC tube }\end{array}$ & 3.5 & 10 & Sheep & Carotid artery & ETE & No & $\begin{array}{l}\text { Microbiological } \\
\text { derived }\end{array}$ & Bacterial cellulose & None & None & None & None & No & 84 & $50 \%$ & $\begin{array}{c}\text { Thrombus formation } \\
\text { next to the proximal } \\
\text { anastomosis }\end{array}$ \\
\hline $\begin{array}{c}\text { Weber } 2017[44] \\
\text { Non-anti-platelet control }\end{array}$ & 4.5 & 10 & Sheep & Carotid artery & ETE & No & $\begin{array}{l}\text { Microbiological } \\
\text { derived }\end{array}$ & Bacterial nanocellulose & None & None & None & None & No & 56 & 0 & NR \\
\hline $\begin{array}{l}\text { Ye } 2012[60] \\
\text { PCL + heparin }\end{array}$ & 2 & 4 & Dog & Femoral artery & ETE & No & Synthetic & PCL & Heparin & None & None & None & No & 28 & $100 \%$ & No failure \\
\hline $\begin{array}{l}\text { Zhao } 2010[01] \\
\text { 2 months }\end{array}$ & 3 & 4 & Sheep & Carotid artery & ETE & No & $\begin{array}{l}\text { Autologous cells on } \\
\text { allograft }\end{array}$ & $\begin{array}{l}\text { Decellularized ovine carotid } \\
\text { artery }\end{array}$ & None & Autologous & $\begin{array}{l}\text { MSCS } \\
\text { differentiated } \\
\text { ECs-like cells }\end{array}$ & $\begin{array}{c}\text { MSCs } \\
\text { differentiated } \\
\text { SMCs-like cells }\end{array}$ & No & 60 & $100 \%$ & No failure \\
\hline $\begin{array}{l}\text { Zhao } 2010 \text { [61] } \\
5 \text { months }\end{array}$ & 3 & 4 & Sheep & Carotid artery & ETE & No & $\begin{array}{l}\text { Autologous cells on } \\
\text { allograft }\end{array}$ & $\begin{array}{l}\text { Decellularized ovine carotid } \\
\text { artery }\end{array}$ & None & Autologous & $\begin{array}{l}\text { MSCs } \\
\text { differentiated } \\
\text { ECS-like cells }\end{array}$ & $\begin{array}{c}\text { MSCS } \\
\text { differentiated } \\
\text { SMCs-like cells }\end{array}$ & No & 150 & $100 \%$ & No failure \\
\hline $\begin{array}{l}\text { Zhao } 2010[61]] \\
\text { Acellular control }\end{array}$ & 3 & 4 & Sheep & Carotid artery & ETE & No & Allogeneic & $\begin{array}{l}\text { Decellularized ovine carotid } \\
\text { artery }\end{array}$ & None & None & None & None & No & 14 & 0 & Thrombus \\
\hline
\end{tabular}

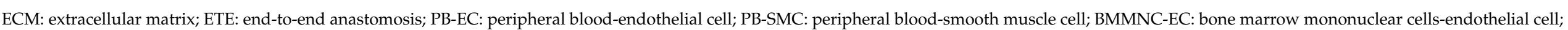

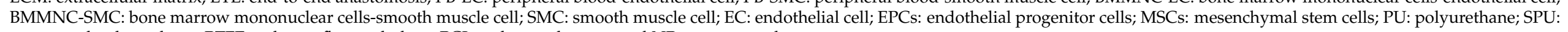
segmented polyurethane; PTFE: polytetrafluoroethylene; PCL: polycaprolactone; and NR: not reported. 
Table 6. SD-TEVGs evaluated in large animals in arterial bypass using end-to-end (ETE) anastomosis (with antithrombotic therapy).

\begin{tabular}{|c|c|c|c|c|c|c|c|c|c|c|c|c|c|c|c|c|}
\hline \multirow[t]{2}{*}{ Study Group } & \multicolumn{6}{|c|}{ Model } & \multicolumn{3}{|c|}{ Graft } & \multicolumn{3}{|c|}{ Modification } & & \multicolumn{3}{|c|}{ Outcome } \\
\hline & $\mathrm{D}(\mathrm{mm})$ & $\stackrel{\mathrm{L}}{\mathrm{cm})}$ & Animal & $\begin{array}{l}\text { Implantation } \\
\text { site }\end{array}$ & Anastomosis & $\begin{array}{l}\text { Antithro- } \\
\text { mbotic } \\
\text { therapy }\end{array}$ & Graft type & Material & $\begin{array}{l}\text { Chemical } \\
\text { Modification }\end{array}$ & $\begin{array}{c}\text { Biological } \\
\text { modification }= \\
\text { Recellulariza- } \\
\text { tion }\end{array}$ & Luminal cell type & $\begin{array}{l}\text { Medial } \\
\text { cell type }\end{array}$ & $\begin{array}{l}\text { Mechanical } \\
\text { modification = } \\
\text { Precondition }\end{array}$ & $\begin{array}{l}\text { Follow-up } \\
\text { (day) }\end{array}$ & Patency & Graft Failure \\
\hline $\begin{array}{l}\text { Arts 2002 [62] } \\
\text { Transduction } 3 \text { weeks }\end{array}$ & 4 & 5 & Dog & Carotid artery & ETE & Yes & Synthetic & ePTFE & None & Autologous & $\begin{array}{c}\text { Fat-derived } \\
\text { microvascular } \\
\text { endothelial cells }\end{array}$ & None & No & 21 & $100 \%$ & No failure \\
\hline $\begin{array}{l}\text { Arts } 2002[62] \\
1 \text { month }\end{array}$ & 4 & 5 & Dog & Carotid artery & ETE & Yes & Synthetic & ePTFE & None & Autologous & $\begin{array}{c}\text { Fat-derived } \\
\text { microvascular } \\
\text { endothelial cells }\end{array}$ & None & No & 30 & $83 \%$ & Thrombus \\
\hline $\begin{array}{l}\text { Arts } 2002[62] \\
1 \text { month control }\end{array}$ & 4 & 5 & Dog & Carotid artery & ЕTE & Yes & Synthetic & ePTFE & None & None & None & None & No & 30 & $83 \%$ & Thrombus \\
\hline $\begin{array}{l}\text { Arts } 2002[62] \\
12 \text { months }\end{array}$ & 4 & 5 & Dog & Carotid artery & ETE & Yes & Synthetic & ePTFE & None & Autologous & $\begin{array}{c}\text { Fat-derived } \\
\text { microvasular } \\
\text { endothelial cells }\end{array}$ & None & No & 365 & $100 \%$ & No failure \\
\hline $\begin{array}{l}\text { Chue 2004 [63] } \\
\text { no mesh }\end{array}$ & 3.75 & 6 & Dog & Femoral artery & ETE & Yes & $\begin{array}{l}\text { Autologous cells on } \\
\text { autologous ECM }\end{array}$ & $\begin{array}{l}\text { IBTA (from peritoneal and } \\
\text { pleurl cavities, based on } \\
\text { Polyethylene or C-flex) }\end{array}$ & None & None & None & None & No & 90-195 & $83 \%$ & Organized thrombus \\
\hline $\begin{array}{l}\text { Chue 2004 [63] } \\
\text { PGA mesh }\end{array}$ & 3.75 & 6 & Dog & Femoral artery & ETE & Yes & $\begin{array}{l}\text { Autologous cells on } \\
\text { autologous ECM } \\
\text { and synthetic graft }\end{array}$ & $\begin{array}{l}\text { IBTA with biodegradable } \\
\text { PGA mesh (from peritoneal } \\
\text { and pleural cavities, based } \\
\text { on Polyethylene) } \\
\text { IBTA with) }\end{array}$ & None & None & None & None & No & 90-195 & $75 \%$ & Organized thrombus \\
\hline $\begin{array}{l}\text { Chue 2004 [63] } \\
\text { polypropylene mesh }\end{array}$ & 3.75 & 6 & Dog & Femoral artery & ETE & Yes & $\begin{array}{l}\text { Autologous cells on } \\
\text { autologous ICM } \\
\text { and synthetic graft }\end{array}$ & $\begin{array}{l}\text { nonbiodegradable } \\
\text { polypropplene mesh (from } \\
\text { peritoneal and pleural } \\
\text { cavities, based on } \\
\text { Polyethylene) }\end{array}$ & None & None & None & None & No & 90-195 & 0 & Organized thrombus \\
\hline $\begin{array}{c}\text { Ju } 2017 \text { [64] } \\
\text { Acellular control }\end{array}$ & 4.75 & 5 & Sheep & Carotid artery & ETE & Yes & $\begin{array}{l}\text { Synthetic and } \\
\text { natural }\end{array}$ & $\begin{array}{l}\text { Bilayered blending of PCL } \\
\text { and calf type I collagen }\end{array}$ & None & None & None & None & No & 10 & 0 & Thrombus \\
\hline $\begin{array}{l}\text { Kaushal 2001 [65] } \\
130 \text { days }\end{array}$ & 4 & 4.5 & Sheep & Carotid artery & ETE & Yes & $\begin{array}{c}\text { Autologous cells on } \\
\text { xenograft }\end{array}$ & $\begin{array}{l}\text { Decellularized porcine iliac } \\
\text { blood artery }\end{array}$ & None & Autologous & PB-EC & None & Yes & 130 & $100 \%$ & No failure \\
\hline $\begin{array}{l}\text { Kaushal 2001 [65] } \\
\text { Acellular control }\end{array}$ & 4 & 4.5 & Sheep & Carotid artery & ETE & Yes & Xenogeneic & $\begin{array}{l}\text { Decellularized porcine iliac } \\
\text { blood artery }\end{array}$ & None & None & None & None & No & 15 & $25 \%$ & Thrombus \\
\hline 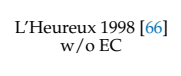 & 3 & 5 & Dog & Femoral artery & $\begin{array}{c}\text { ETE } \\
\text { (interpreted } \\
\text { from figure) }\end{array}$ & $\begin{array}{l}\text { Yes, immuno- } \\
\text { suppression }\end{array}$ & Xenogeneic & $\begin{array}{l}\text { Dehydrated Human } \\
\text { vascular SMC and } \\
\text { fibroblasts cells sheet }\end{array}$ & None & None & None & None & No & 7 & $50 \%$ & Thrombus \\
\hline $\begin{array}{l}\mathrm{Ma} 2017 \text { [67] } \\
\text { DAFP + EC }\end{array}$ & $\begin{array}{l}4 \text { (outer } \\
\text { diameter) }\end{array}$ & 6 & Dog & Carotid artery & ETE & Yes & $\begin{array}{l}\text { Autologous cells on } \\
\text { xenograft }\end{array}$ & $\begin{array}{l}\text { Decellularizized aortae of } \\
\text { fetal pigs }\end{array}$ & None & Autologous & Vein-EC & None & Yes & 180 & $100 \%$ & No failure \\
\hline $\begin{array}{l}\text { Mrowcynnski } 2014 \\
\text { ePTFE control }\end{array}$ & 4 & 5 & Pig & Carotid artery & ETE & Yes & Synthetic & ePTFE & None & None & None & None & No & 28 & $67 \%$ & NR \\
\hline $\begin{array}{l}\text { Mrowcynnki } 2014 \\
\text { If68 } \\
\text { PCL }\end{array}$ & 4 & 5 & Pig & Carotid artery & ETE & Yes & Synthetic & PCL & None & None & None & None & No & 28 & $78 \%$ & $\begin{array}{l}\text { Thrombus from } \\
\text { prosthetic kink }\end{array}$ \\
\hline $\begin{array}{l}\text { Neff 2011 [69] } \\
\text { dsTEBV (EC + SMC) }\end{array}$ & 5 & 6 & Sheep & $\begin{array}{l}\text { Carotid artery } \\
\text { or femoral } \\
\text { artery }\end{array}$ & ETE & Yes & $\begin{array}{c}\text { Autologous cells on } \\
\text { xenograft }\end{array}$ & $\begin{array}{l}\text { Decellularized porcine } \\
\text { carotid arterial segments }\end{array}$ & None & Autologous & PB-EC & $\begin{array}{l}\text { Artery- } \\
\text { SMC }\end{array}$ & Yes & 120 & $100 \%$ & No failure \\
\hline $\begin{array}{l}\text { Neff 2011 [69] } \\
\text { ecCEBV (EC) }\end{array}$ & 5 & 6 & Sheep & $\begin{array}{c}\text { Carotid artery } \\
\text { or femoral } \\
\text { artery }\end{array}$ & ETE & Yes & $\begin{array}{c}\text { Autologous cells on } \\
\text { xenograft }\end{array}$ & $\begin{array}{l}\text { Decellularized porcine } \\
\text { carotid arterial segments }\end{array}$ & None & Autologous & PB-EC & None & Yes & 120 & $100 \%$ & No failure \\
\hline $\begin{array}{l}\text { Nemcova } 2001[701 \\
\text { Acellular xenograft }\end{array}$ & 4 & 5 & Dog & Femoral artery & ETE & Yes & Xenogeneic & $\begin{array}{l}\text { Acellular porcine small } \\
\text { intestinal submucosa }\end{array}$ & $\begin{array}{l}\text { Type I } \\
\text { boine } \\
\text { collagen }\end{array}$ & None & None & None & No & 63 & $89 \%$ & Wall thickening \\
\hline
\end{tabular}


Table 6. Cont

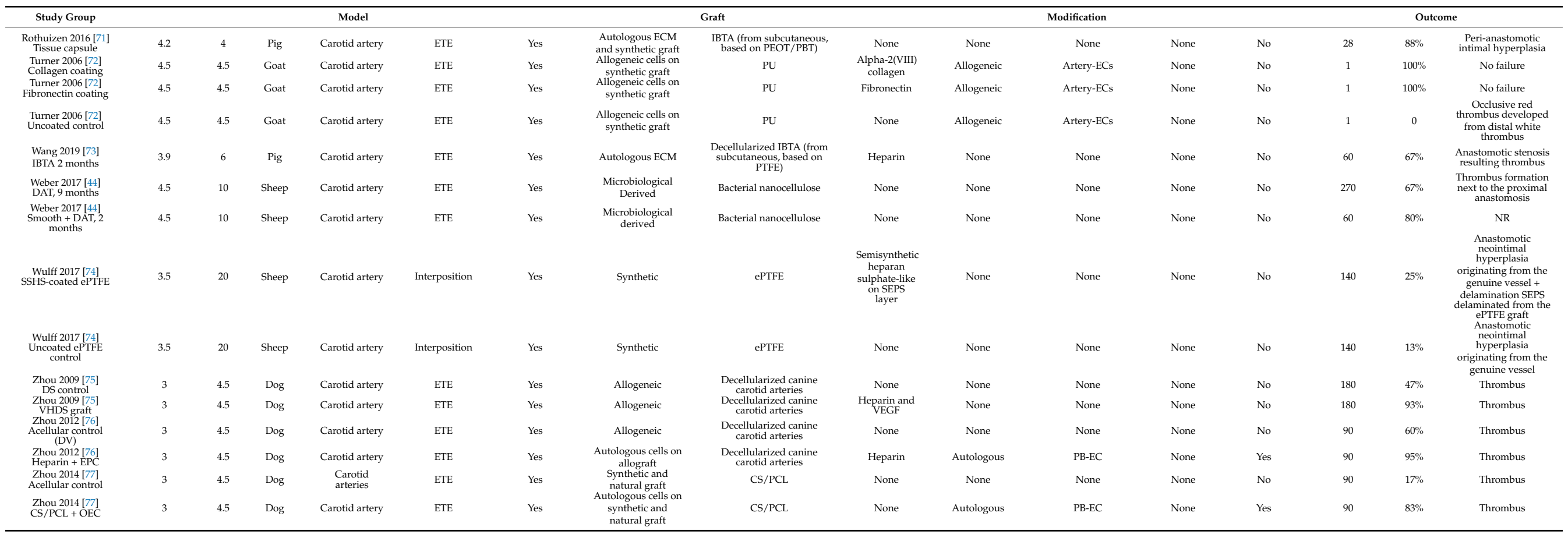

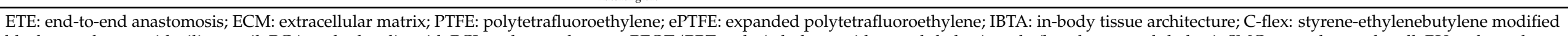

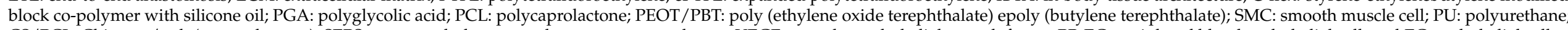

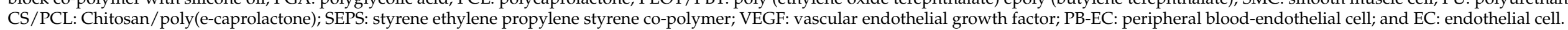


Table 7. SD-TEVGs evaluated in large animal in arteriovenous shunt.

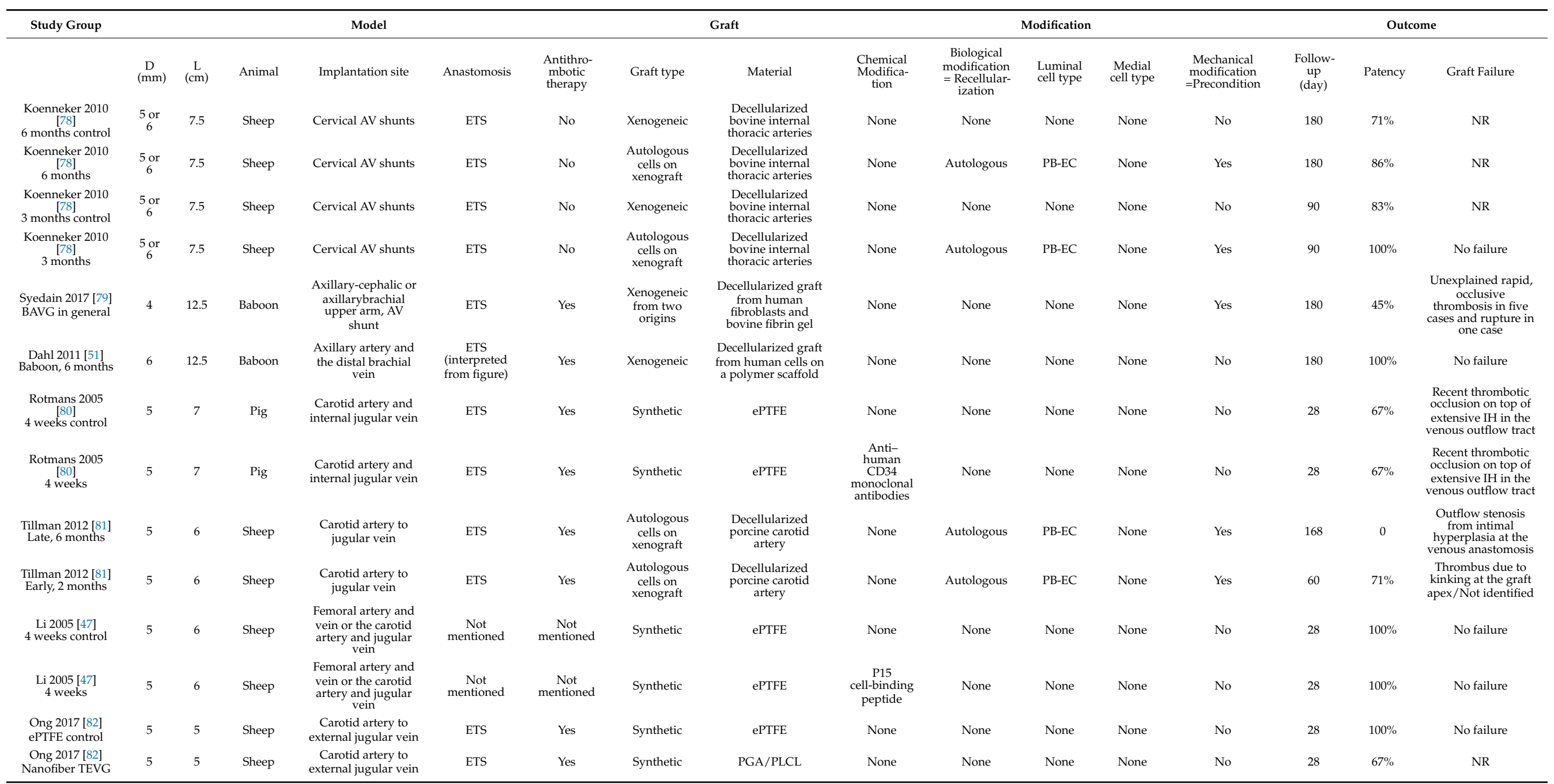


Table 7. Cont.

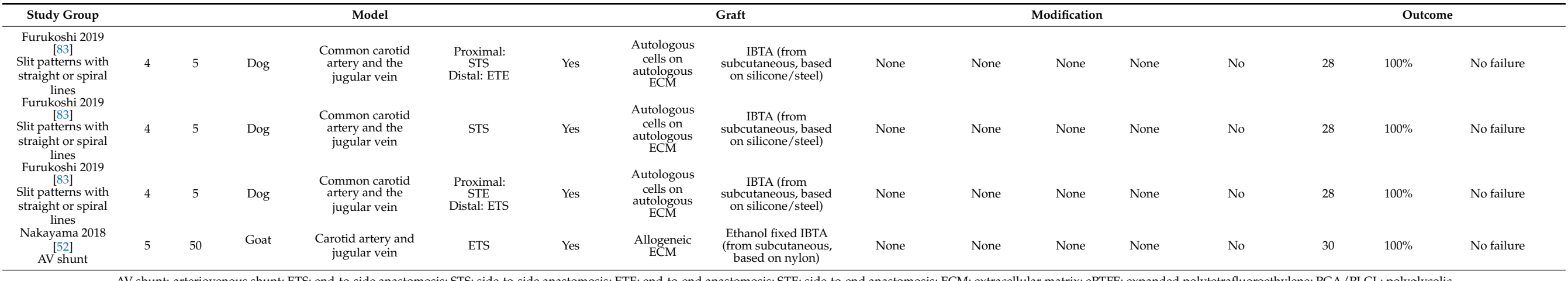

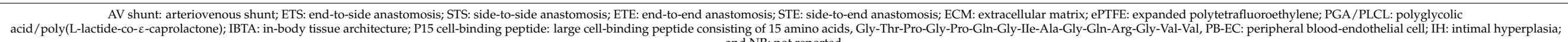
and
and NR: not reported. 
Table 8. Fabrication methods of SD-TEVG.

\begin{tabular}{|c|c|c|c|}
\hline Graft Type & Material & Fabrication & Reference \\
\hline Synthetic & $\begin{array}{l}\text { Dacron, ePTFE, PU, PCL, PLCL, PGA, } \\
\text { PLA, PLLA, PLGA, PGS, PEUU }\end{array}$ & $\begin{array}{l}\text { Electrospinning, molding, 3D Printing, laser } \\
\text { degradation, hydrogel }\end{array}$ & $\begin{array}{c}{[45,47,53,58,60,62,68,} \\
72,74,80,82]\end{array}$ \\
\hline \multirow{3}{*}{ Natural } & $\begin{array}{l}\text { Collagen, elastin, fibrin, hyaluronic } \\
\text { acid, silk fibroin, gelatin, chitosan }\end{array}$ & $\begin{array}{l}\text { Electrospinning, molding, rolling, 3D Printing, } \\
\text { laser degradation, hydrogel }\end{array}$ & {$[44,54,59]$} \\
\hline & Cell-secreted ECM & Hydrogel, rolling, self-assembled cell sheets & {$[66,79]$} \\
\hline & Native ECM & $\begin{array}{l}\text { Decellularization or crosslinking of native } \\
\text { tubular organs (vessels, ureters and small } \\
\text { intestinal submucosa) }\end{array}$ & $\begin{array}{l}{[46,49,50,55,58,61,65,} \\
67,69,70,75,76,78,81]\end{array}$ \\
\hline Hybrid & Combination of above & $\begin{array}{l}\text { Combination of above, e.g., in-body tissue } \\
\text { architecture (IBTA)/ in vivo tissue engineering }\end{array}$ & $\begin{array}{c}{[51,52,56,57,63,64,71,} \\
73,77,83]\end{array}$ \\
\hline
\end{tabular}

Dacron: Polyethylene terephthalate (PET); ePTFE: expanded polytetrafluoroethylene; PU: Polyurethane; PCL: polycaprolactone; PLCL: poly(L-lactide-co- $\varepsilon$-caprolactone); PGA: polyglycolic acid; PLA: poly-lactic acid; PLLA: poly-l-lactic acid; PLGA: poly (lactide-co-glycolide); PGS: Poly(glycerol-sebacate); PEUU: poly(ether urethane urea); and ECM: extracellular cellular matrix.

\subsubsection{Synthetic SD-TEVGs}

Synthetic SD-TEVGs have been extensively tested likely due to their easy availability and customization (Table 9).

Table 9. Advantages and disadvantages of SD-TEVGs in different material.

\begin{tabular}{|c|c|c|}
\hline SD-TEVG Type & Advantages & Disadvantages \\
\hline Synthetic SD-TEVGs & - Easy availability and customization. & - Biological incompatibility and thrombogenicity. \\
\hline Natural SD-TEVGs & $\begin{array}{l}\text { - Low immunogenicity and high } \\
\text { biological compatibility (allogeneic). } \\
\text { Adequate mechanical strength (native } \\
\text { ECM-based). }\end{array}$ & $\begin{array}{l}\text { - } \quad \text { Immunogenicity and thrombogenicity (xenogeneic) } \\
\text { - Weakness in mechanical strength (natural } \\
\text { - } \quad \text { Limited availability (native ECM-based). }\end{array}$ \\
\hline Hybrid SD-TEVGs & $\begin{array}{l}\text { - } \quad \text { Biological compatibility } \\
\text { - } \quad \text { Adequate mechanical strength } \\
\text { - } \quad \text { Availability }\end{array}$ & $\begin{array}{l}\text { - Thrombogenicity when incorporated with } \\
\text { xenogeneic natural polymers. } \\
\text { - Short off-shelf time and unavailability for acute } \\
\text { procedures. }\end{array}$ \\
\hline
\end{tabular}

Conventional prosthetic vascular grafts like Dacron and expanded polytetrafluoroethylene (ePTFE) are biostable grafts, which can prevent mechanical deformation in high-pressure and high-flow-rate arteries, like the aorta. Thus, they are safe grafts to be used in large diameter bypass grafting and offer satisfying patency rates and outcomes. However, they fail when applied in small-diameter bypass grafting, since they result in thrombus formation and do not facilitate endothelialization when uncoated [84]. They are also very rigid and do not match the compliance of the native vessel [85-87]. Considering the safe and positive experience when applying ePTFE as implants in patients, ePTFE therefore often serve as negative controls when evaluating other novel synthetic SD-TEVGs. In the studies screened by our systematic search (Tables 4-7), 11 out of 39 studies have examined synthetic SD-TEVGs. Eight studies included SD-TEVGs made of pure ePTFE without any modification as a negative control group, four of which served as control for other four types of grafts (Tables 4-7) $[53,58,68,82]$ and four of which served as a scaffold for testing the effect of surface modifications (Tables 6 and 7) $[47,62,74,80]$. Pure ePTFE grafts displayed a patency of $67-100 \%$ after about 1 month in five studies $[47,62,68,80,82]$. Four of these studies used systemic antithrombotic treatment, while one study did not refer to such treatment [47]. When the follow-up period increased to 140-365 days, the patency of ePTFE decrease to $0-50 \%$ even in the presence of systemic antithrombotic treatment $[53,62,74]$. The only study that examined pure ePTFE without systemic antithrombotic treatment revealed poor patency $(0 \%)$ after 7 days [58]. Thus, pure ePTFE is inapplicable as a material for 
SD-TEVGs due to its poor patency resulting from high thrombogenicity [53] or late intimal hyperplasia $[74,80]$. These events are well known causes for graft failure and may reflect resistance of a hydrophobic surface to the endothelial cell (ECs), compliance mismatch, or prolonged foreign body reaction to the polymer [86,88-90].

Polyurethane (PU), another synthetic material based on advanced material techniques, has been produced as both biostable and biodegradable grafts [91] with compliance matching the native vessel [92]. Since biodegradable PU grafts show better outcomes, biodegradable synthetic polymers have attracted more and more attention in the field and are extensively investigated for developing SD-TEVGs. Recently explored biodegradable synthetic polymers include, but are not limited to, polycaprolactone (PCL), poly(L-lactideco- $\varepsilon$-caprolactone) (PLCL), polyglycolic acid (PGA), poly-lactic acid (PLA), poly-l-lactic acid (PLLA), poly (lactide-co-glycolide (PLGA), poly (glycerol-sebacate) (PGS), and poly(ether urethane urea) (PEUU) [93-99]. Biodegradable synthetic vascular grafts have already been investigated in humans, and the first trial comprised a TEVG (diameter: $10 \mathrm{~mm}$ ) consisting of PCL and PLA copolymers strengthened by woven PGA, which was then seeded with autologous venous patient cells and implanted into a 4-year-old patient for pulmonaryartery reconstruction by Shin'oka et al. in 2001 [100]. The same group further evaluated similar TEVGs seeded with bone marrow cells as large diameter conduits in patients with congenital heart disease for midterm and long-term functionality in 2005 and 2010 [101,102]. Likewise, PCL-based large-diameter vessel grafts have been implanted into patients with congenital heart disease [103], whereas PLLA has been tested for right ventricular outflow tract (RVOT) reconstruction [104]. However, all these clinical trials were conducted in large-diameter vessel sites [100-103], and still no small-diameter biodegradable synthetic vascular graft has been tested in humans, despite that the idea of using biodegradable material to support tissue reconstruction was presented decades ago [105]. By contrast, the retrieved large-animal studies reveal that several biodegradable synthetic polymers, including PU [53,72], PGA/PLCL [82] and PCL [45,60,68], have been examined as smalldiameter vessel grafts in large-animal studies with clinically relevant length/diameter ratios. In this regard, Turner et al. reported that, already within $24 \mathrm{~h}$, PU grafts were prone to occlusive red thrombus, developed from distal white thrombus, although the graft was endothelialized before implantation into goat carotid artery [72]. The authors noted only an $8 \%$ retention of seeded ECs on the PU grafts after exposure to high blood flow in vivo, which indicates a high thrombogenicity of PU grafts. However, the ECs seeded were from allogeneic origin, which may harm the retention of cells and in the end lower the patency. As compared to the synthetic PU polymer, natural alpha-2 (VIII) collagen or fibronectin polymer displayed enhancement in affinity for ECs when coated to the PU graft, with about 50\% retention of ECs after exposure to high blood flow in vivo [72]. Interestingly, Soldani et al. also evaluated a PU graft composed of poly(ether)urethanepolydimethylsiloxane (PEtU-PDMS), a polymer belonging to the large PU family, and reported an excellent long-term patency at 100\% after 2 years in sheep carotid bypass with 1 month postoperation antithrombotic treatment using aspirin [53], which is reported to fail in inhibiting platelet aggregation in sheep [106,107]. Thus, these two PU grafts display converse in vivo outcome. Besides PU, another polymer: PGA/PLCL has been involved in one retrieved study (Table 7). Ong et al. implanted PGA/PLCL grafts in an arteriovenous shunt model and presented $67 \%$ patency under systemic antithrombotic treatment after 28 days, which is lower than what has been observed in ePTFE controls (100\%) [82]. As compared to other polymers, studies have shown favorable mechanical properties, biocompatibility, and appropriate biodegradability of PCL-based SD-TEVGs $[88,108,109]$. In agreement, we have recently shown that PCL scaffolds may be easily recellularized with adipose-derived regenerative cells (ADRCs) during in vitro static culture in a dish, as well as in dynamic culture using a 3D bioreactor with pulsatile media flow [45]. Specifically, we found vigorous proliferation of ADRCs in the PCL material in combination with complete ADRC migration throughout the scaffold under both static and dynamic conditions. These findings thus underscore the high cytocompatibility of a PCL-based SD-TEVGs. However, 
Mrowczynski et al. (Table 6) have previously reported a transprosthetic blood leakage of PCL grafts shortly after reconstruction of blood flow in bypass, whereas a late thrombotic occlusion resulted from a prosthetic kink [68]. To avoid such in vivo complications, we designed our PCL graft with a lower porosity ( 60 versus $80 \%$ ) and a reduced thickness (250 versus $500 \mu \mathrm{m})[45,68]$. Indeed, our in vivo results, when testing the PCL-based SD-TEVGs as sheep carotid substitution, suggested that transprosthetic blood leakage and prosthetic kink were successfully prevented [45]. Moreover, our PCL vessel graft (Table 4) displayed easy surgical handling, secured anastomosis, and absence of any rupture, aneurysm, or necrosis within the follow-up period, which further emphasizes its appropriate and stable mechanical properties and biodegradation. It is likely that our PCL is prevented from excessive degradation that would otherwise weaken the mechanical strength and lead to graft rupture or dilation [110]. Besides Mrowczynski's [68] and our study [45], one other study (Tables 4-6) has investigated PCL in arterial bypass substitution [60]. Whereas Ye et al. conjugated heparin to the PCL graft and revealed a 100\% patency after 4 weeks, Mrowczynski et al. demonstrated a 78\% patency for PCL grafts without any conjugation but under systemic antithrombotic treatment after 4 weeks $[60,68]$. Thus, PCL-based grafts may maintain patency in a short period either under local conjugation or systemic administration of antithrombotic treatment. This is clearly supported by our study, showing that daily use of antithrombotic treatment increases graft patency [45]. Thus, similar to the PCL grafts in Mrowczynski and Ye's studies [60,68], our PCL grafts (Table 4) exhibited 100\% patency after 28 days with the daily use of antithrombotic treatment, whereas the PCL grafts without antithrombotic treatment displayed very poor patency $(0 \%)$ already in the first week after implantation [45]. Thus, it seems that PCL-based SD-TEVGs are highly thrombogenic, but this may be significantly reduced by systemic antithrombotic treatment and recellularization as discussed below.

In general, the biodegradable synthetic SD-TEVGs evaluated in vivo in large-animal models manifest robust and stable mechanical properties required for in vivo hemodynamics. However, very limited types of synthetic SD-TEVGs have come to the large-animal evaluation, and their thrombogenicity and biocompatibility require substantial improvement through either antithrombotic treatment or modification on the grafts. Moreover, considering most studies included in the large-animal studies only reported short-term ( $<28$ days) results, longer follow-up in vivo is valuable and thus needed for understanding the medium- and long-term outcome and interaction mechanisms between this polymer and host environment.

\subsubsection{Natural SD-TEVGs}

Another popular type of materials are those polymers and matrix naturally present in humans and animals or generated by cells or even microorganisms (Figure 1). Natural SD-TEVGs manifest low immunogenicity, high biocompatibility, and an inherent biodegradability, which suppress the immune response and facilitate cell growth and tissue regeneration (Table 9). Nineteen out of 39 retrieved large-animal studies (Tables 4-7) have evaluated natural SD-TEVGs. Three out of these 19 studies have used natural polymerbased SD-TEVGs $[44,54,59]$, whereas two studies investigated cell-secreted extracellular cellular matrix (ECM)-based SD-TEVGs [66,79], and the 14 remaining used native ECMbased SD-TEVGs (Table 8).

\subsubsection{Natural Polymer-Based SD-TEVGs}

Natural polymers including collagen, elastin, fibrin, hyaluronic acid, silk fibroin, gelatin, and chitosan [111] can be artificially constructed into TEVGs by fabrication methods like electrospinning, molding, rolling, 3D printing, laser degradation, and hydrogel (Figure 1 and Table 8). Collagen and elastin are especially interesting molecules [112,113], which are components of the ECM in native blood vessels. In this regard, Aper et al. reported a SD-TEVG, based on a highly compact fibrin matrix, with a $100 \%$ patency after 180 days when the surface was coated with Factor XIII and recellularized by both ECs and 
smooth muscle cell (SMCs) before implantation [54]. However, graft rupture, which is a typical failure due to weak mechanical strength and fast natural polymer degradation, was noted in the 30 day observation group. Scherner's group developed another type of SD-TEVGs with natural polymers, composed of a microbiological derived matrix called bacterial nanocellulose (BNC). They developed a first [59] and second [44] generation, where the main difference concerned the wall thickness (2.0-3.5 mm versus $1.0-2.5 \mathrm{~mm}$, respectively). First-generation BNCs displayed no graft rupture due to their high mechanical strength, but $50 \%$ failed due to thrombus formation next to the proximal anastomosis after 84 days without any thrombotic treatment [59]. By contrast, second-generation BNC grafts demonstrated $0 \%$ patency after 56 days without dual antiplatelet treatment [44], thus even worse than the first-generation BNC grafts. However, the patency of second-generation BNC grafts may be rescued by dual antiplatelet treatment (67\% patency after 270 days) and surface smoothing ( $80 \%$ patency after 60 days) as shown by Weber et al. [44].

\subsubsection{Cell-Secreted ECM-Based SD-TEVGs}

Like natural polymer-based SD-TEVGs, cell-secreted ECM-based SD-TEVGs are constructed by a similar fabrication method but involve cultivation of cells (Figure 1 and Table 8). L'Heureux et al. have generated a three-layered graft consisting of human fibroblast and SMC sheets, which has a mechanical strength close to that found in human vessels [66]. When the grafts were dehydrated and evaluated in large-animal models, the patency was $50 \%$ after 7 days with grafts failing from thrombus formation [66]. These multisheeted grafts are fabricated by rolling of self-assembled cell sheets where the resulting vascular graft consists of ECM produced by in vitro cultured cells, with the nuclei and cell cytoplasm being removed afterwards by dehydration [33,66]. Indeed, L'Heureux's group have evaluated such grafts as arteriovenous shunts in human (Table 2) as discussed above $[31,33,36,37]$. Likewise, by combining both a natural polymer (bovine fibrin gel) and cell-secreted ECM from human fibroblasts, Syedain et al. have generated a natural SD-TEVG that was further preconditioned in a pulsed flow-stretch bioreactor before decellularization. Upon in vivo in baboon, these grafts displayed $45 \%$ patency after 180 days as arteriovenous grafts, where the low patency was caused by graft rupture and thrombus formation [79].

\subsubsection{Native ECM-Based SD-TEVGs}

Finally, native ECM can be obtained from native vessels or other tubular organs from animals and humans to fabricate natural SD-TEVGs by using crosslinking or decellularization procedures (Figure 1 and Table 8) [114]. By these procedures, the ECM and its structure are preserved, and the immunogenicity of native tissues may be eliminated before implantation [115]. Compared to crosslinking, decellularization has gained massive attention in the past decade. Firstly, decellularization removes the immunogenic genetic material in tissue through chemical, enzymatic, or physical approaches [116]. For example, detergents like sodium dodecyl sulfate (SDS) or Triton X-100 and enzymes like DNAse are effective in removing proteins and nucleic acids whereby the immunogenicity is reduced [115]. Secondly, decellularization, if carefully adjusted, preserves the native ECM and their ultrastructure that are thought to benefit stem cell migration into the desired scaffold region and stimulate correct differentiation into functional cell types like endothelial cells and SMCs. Several clinical trials have already shown biocompatibility of decellularized vessels for use in dialysis, heart valves, and vaginal organs [34,117-121].

In the identified large-animal studies (Tables 4-7), 14 studies have evaluated native ECM-based SD-TEVGs, all based on decellularization rather than crosslinking. The involved native organs include carotid artery ( $\operatorname{dog}[55,75,76]$, pig [46,69], sheep [50,61], ostrich [49]), iliac artery (pig [65]), fetal aortae (pig [67]), umbilical artery (human [50]), ureters (dog [58]), and small intestinal submucosa (pig [70]) as arterial bypass grafts, whereas internal thoracic artery (cow [78]) and carotid artery (pig [81]) have been used as grafts for arteriovenous shunts. These SD-TEVGs range in diameter from 3 to $6 \mathrm{~mm}$. In 
arterial bypass models, most xenogeneic native ECM-based SD-TEVGs have turned out to fail due to thrombus within short time $(<15$ days), when no modification like chemical modification or recellularization have been applied to the surface of SD-TEVGs $[49,50,65]$. Decellularized ostrich carotid artery and human umbilical artery grafts display early occlusion ( $<7$ days) from thrombus in xenogeneic sheep models $[49,50]$. By inclusion of antithrombotic treatment, the early thrombotic occlusion ( $<7$ days) of xenogeneic decellularized human umbilical artery may be prevented (unpublished data from our lab). Similarly, Nemcova et al. have evaluated decellularized xenogeneic SD-TEVGs composed of porcine small-intestinal submucosa coated with bovine type I collagen under antithrombotic treatment and obtained a patency of $89 \%$ after 63 days in dog. In vivo failure occurred at 8 weeks due to wall thickening instead of thrombotic occlusion [70]. However, despite the use of antithrombotic treatment, decellularized xenogeneic porcine iliac artery demonstrates only $25 \%$ patency after 15 days due to thrombotic occlusion [65]. Similar to xenogeneic decellularized SD-TEVGs, decellularized allogeneic SD-TEVGs are prone to occlusion as arterial bypass graft without antithrombotic treatment $[46,50,55,58,61]$. Narita et al. estimated that decellularized allogeneic ureters vessel grafts have a $20 \%$ patency after 7 and 56 days, which is similar to that of synthetic ePTFE [58]. Moreover, a similar patency ( $0 \%$ after 14 days [61] and $0 \%$ after 28 days [50]) have been reported for decellularized allogeneic sheep carotid artery. The failure of decellularized allogeneic grafts seems to occur by several mechanisms. Zhao et al. have observed early thrombus in the decellularized sheep carotid artery (dSCA) within 2 weeks [61], whereas we observed intimal hyperplasia rather than thrombus in dSCA after 2-4 weeks [50]. The absence of early thrombus in allogeneic dSCA in our study could be explained by better mechanical match between graft and host artery, since our grafts were harvested from adult sheep and implanted into sheep of the same age, while grafts in Zhao's study were harvested from 6to 8-month-old sheep and implanted into 12-month-old sheep. However, it is also notable that the bodyweight $(57.3-75.6 \mathrm{~kg}$ ) of sheep used in our study is much higher than that $(20-25 \mathrm{~kg})$ used in Zhao's study. The overall diameter of both the dSCA grafts and the host carotid arteries in our study are therefore larger than that in Zhao's study, which may also contribute to the absence of early thrombus in our dSCA grafts [122]. Moreover, without antithrombotic treatment, the decellularized allogeneic canine carotid artery displays a similar poor patency ( $0 \%$ after 14 days) due to thrombus as reported by Cho et al. [55]. The patency of decellularized allogeneic grafts indeed seems to be improved by antithrombotic treatment $[75,76]$. As such, Zhou et al. have shown that decellularized allogeneic canine carotid artery exhibit a patency at $47 \%$ after 180 days and at $60 \%$ after 90 days [75,76], which is substantially higher than the patency for decellularized allogeneic grafts without antithrombotic treatment $[50,55,58,61]$. As also seen with other types of SD-TEVGs, grafts display a higher patency as arteriovenous shunt than as arterial bypass. Accordingly, decellularized xenogeneic bovine internal thoracic arteries used for arteriovenous shunts display 83 and $71 \%$ patency after 90 and 180 days, respectively. The patency was even increased to 100 and $86 \%$ with autologous ECs recellularization [78], which is known in general to improve graft patency [122]. Decellularized xenogeneic porcine carotid arteries with autologous EC recellularization also exhibit relative high mid-term patency ( $71 \%$ after 60 days), which is however substantially deteriorated ( $0 \%$ after 168 days) likely due to intimal hyperplasia induced outflow stenosis at the venous anastomosis [81].

Thus, the large-animal studies clearly reveal that, natural polymer- and cell-secreted ECM-based SD-TEVGs tested in large animals still fail due to a weak strength and thrombogenicity $[44,54,59,66,79]$, whereas decellularized native ECM-based SD-TEVGs possess adequate mechanical strength, since rupture is rarely reported, but their patency remains depending on their origin (xenogeneic or allogeneic) (Table 9). Furthermore, antithrombotic treatment and modifications to the decellularized scaffold such as heparin, or POG7G3REDV peptide conjugation $[49,75]$ or recellularization with autologous cells including their precondition $[58,61,65,69,76,78]$ may significantly improve the patency of decellularized native ECM-based SD-TEVGs. However, availability of the native organ 
source needs to be taken into consideration (Table 9). For instance, arteries from a donor person as exemplified by the carotid artery, iliac artery and internal thoracic artery, tubular organs like ureters and small intestinal submucosa, or fetal tissue like fetal aortae remain scarce in numbers since their isolation requires an available donor and always includes a risk for the donor. Making donor banks from diseased persons would offer an alternative, but the age and associated diseases should be considered. In contrast to this, human umbilical artery is a waste product globally and is thus representing an abundant source without ethical issue.

\subsubsection{Hybrid SD-TEVGs}

Hybrid TEVGs refers to vascular grafts fabricated by combining synthetic and natural molecules or matrix aiming at better performance, since they may bring the advantages of mechanical strength, high biocompatibility, and availability in large-scale production (Table 9). As an example, Nagiah et al. has developed highly compliant SD-TEVGs using synthetic polymers of PU, PCL, or PLA sheathed by gelatine [123]. Not only using a mixture or combination of synthetic and natural materials but also a combination of fabrication methods is getting more and more popular to resolve the challenges with generation of a high complex structure like a small-diameter blood vessel. In-body tissue architecture (IBTA) remains another example, which is also known as in vivo tissue engineering [13]. In 1999, Campbell et al. pioneered this idea by implanting a silastic tube into the peritoneal or pleural cavity of dogs to fabricate autologous SD-TEVGs [63,124]. The rationale for this method to be suggested, is that it implicates the anticoagulant ability of mesothelial cells [125] and the foreign body reaction to biomaterials [126]. Nowadays, this idea is still being explored though with biodegradable synthetic material being implanted for development within a subcutaneous cavity instead of the peritoneal or pleural cavity $[73,83,127,128]$. As for the large-animal studies (Tables 4-7), 10 studies have evaluated hybrid SD-TEVGs as arterial bypass grafts $[51,52,56,57,63,64,71,73,77,83]$ and arteriovenous shunts $[51,52,83]$.

\subsubsection{In-Vitro-Developed Hybrid SD-TEVGs}

Five of the above 10 mentioned studies evaluated the hybrid SD-TEVGs constructed in vitro by blending polymer or cells (Figure 1) [51,56,57,64,77]. As such, Dahl et al. engineered SD-TEVGs by growing allogeneic SMCs on rapidly degraded PGA tubular grafts followed by decellularization [51]. The hybrid SD-TEVGs were further seeded with autologous ECs and evaluated in both carotid and coronary-artery bypass. Although a very distinguished coverage (0-60\%) of ECs was achieved, most of the grafts (83\%) maintained patency after a follow-up from 7 to 365 days, which may both be attributed to systematic antithrombotic treatment and also the small numbers of residing ECs. Similarly, He et al. has grown autologous SMCs in a type I collagen gel which was then wrapped in segmented PU film as an outer layer. The SD-TEVGs were seeded with autologous ECs and displayed $100 \%$ patency after 180 days in canine carotid artery bypass with confluent coverage of ECs, even without applying systemic antithrombotic treatment [56]. A similar hybrid graft composed of collagen I and wrapping segmented PU film manifested similar patency (100\% after 90 days) in vivo although SMCs were not blended in during fabrications, which suggests that the mechanical strength of these segmented PU-based hybrid SD-TEVGs derived mainly from the PU film rather than the SMCs and the secreted ECM from cells [57]. Aside from PU-based hybrid SD-TEVGs, two studies have developed PCL-based hybrid SD-TEVGs. As such, Zhou et al. developed hybrid SD-TEVGs by blending chitosan with PCL and found a poor patency of $17 \%$ after 90 days [77], and similarly Ju et al. reported zero patency for hybrid SD-TEVGs composed by type I collagen and PCL after 10 days [64], both as carotid arterial bypass grafts under antithrombotic treatment $[64,77]$. This contrasts the relative high patency of PCL synthetic SD-TEVGs under antithrombotic treatment observed by Mrowczynski [68] and us [45] and might be explained by the incorporation of xenogeneic natural polymers that are known to accelerate blood clotting, like collagen [64] 
or chitosan [77], into the SD-TEVGs. In specific, type I collagen is the dominant collagen in human arteries and a strong trigger for platelet aggregation and thrombosis [129], and similarly chitosan has been shown to have hemostatic effects [130]. Even so, a significant increased patency (100\% after 100 days) was seen when preconditioned autologous ECs and SMCs were seeded on type I collagen/PCL hybrid SD-TEVGs [64], and likewise an improved patency ( $83 \%$ after 90 days) was reported for autologous ECs seeded chitosan/PCL hybrid SD-TEVGs [77]. Thus, to further evaluate the contribution to in vivo patency from the improved affinity between ECs and the natural type I collagen or chitosan polymers of PCL-based hybrid SD-TEVGs, more large-animal studies evaluating endothelialized PCLbased hybrid SD-TEVGs are needed. Neverthless, in-vitro-developed hybrid SD-TEVGs indeed seem to have favorable patency when seeded with endothelial cells $[51,56,57,64,77]$. However, as unseeded hybrid grafts $[64,77]$, they exhibit very poor patency despite the use of antithrombotic treatment, likely due to the incorporation of xenogeneic natural polymers as discussed above. This is similar to what has been observed for xenogeneic native ECM-based SD-TEVGs [65], indicating that the xenogeneic originated molecules may harm the graft outcome (Table 9), but this can be rescued by endothelialization for an intermediate period.

\subsubsection{IBTA-Based SD-TEVGs}

As compared to the above mentioned in-vitro-developed hybrid SD-TEVGs, IBTAbased SD-TEVGs utilize the host environment as an in vivo bioreactor to initiate the foreign body reaction against synthetic tubing mold and engineer ECM (Figure 1) [126]. The low immunogenicity of IBTA-based SD-TEVGs is a clear advantage, but the off-shelf time and unavailability for acute procedures are disadvantages (Table 9). Currently, five studies have evaluated IBTA-based SD-TEVGs in vivo in large animals (Tables 4-7), and all these studies applied the use of systemic antithrombotic treatment $[52,63,71,73,83]$. For instance, Chue et al. inserted polyethylene tubing, either bare or wrapped in a biodegradable PGA mesh or a nonbiodegradable polypropylene (Prolene) mesh, in the peritoneal or pleural cavity of dogs for three weeks and then harvested autologous IBTA-based SD-TEVGs with myofibroblasts [63]. After implantation as femoral artery bypass grafts for 90 to 195 days, the patency was $83 \%, 75 \%$ and $0 \%$ for bare polyethylene, wrapped in PGA mesh or polypropylene mesh, respectively. The grafts failed due to thrombus regardless the material of synthetic tubing mold inserted and the use of antithrombotic treatment. Similarly, Rothuizen et al. inserted a rod composed of copolymers (PEOT/PBT) that was wrapped by an external PCL sheet subcutaneously for 4 weeks [71]. The obtained autologous IBTA-based SD-TEVGs manifested $88 \%$ patency after 28 days as carotid artery grafts, and the failure of the grafts was attributed to peri-anastomotic intimal hyperplasia, which is different from that reported by Chue and coworkers [63]. Moreover, Wang et al. inserted the PTFE rod subcutaneously and generated autologous IBTA-based SD-TEVGs, which were further decellularized and coated with heparin [73]. These decellularized autologous IBTAbased SD-TEVGs, used as carotid artery bypass grafts, were reported to have a comparable patency ( $67 \%$ after 60 days) to the abovementioned non-decellularized IBTA-based SDTEVGs [63,71]. Still, grafts failed due to anastomotic stenosis and resulting thrombus, but exhibited excellent mechanical strength in vivo. Mechanical stability is also observed by Furukoshi et al., that the subcutaneously generated autologous IBTA-based SD-TEVGs manifested $100 \%$ patency and no aneurysm formation or hemorrhage as arteriovenous shunt within the 28 days follow-up even with repeat percutaneous puncture [83]. Besides the autologous IBTA-based SD-TEVGs, Nakayama et al. developed allogeneic IBTA-SDTEVGs by inserting nylon mold subcutaneously and decellularized with $70 \%$ ethanol [52]. Similar to the autologous IBTA grafts, the allogeneic IBTA-based SD-TEVGs fixed by ethanol showed favorable patency ( $100 \%$ patency after 30 days), both as carotid artery bypass grafts and arteriovenous shunts with application of antithrombotic treatment.

Thus overall, as arterial grafts, IBTA-based SD-TEVGs [52,63,71,73], which are from allogeneic [52] and autologous $[63,71,73]$ origins, display relative higher patency than in- 
vitro-developed hybrid SD-TEVGs $[64,77]$ that contain xenogeneic natural polymers, when both used with antithrombotic treatment and no endothelial recellularization. This suggests that the nonxenogeneic origin of ECM is important to improve the in vivo patency of SDTEVGs. Nevertheless, it may also indicate that myofibroblasts transdifferentiated into SMCs have an impact $[63,124]$. Since current IBTA-based SD-TEVGs lack EC seeding $[52,63,71,73]$, it would be of great interests to investigate if EC seeding may improve in vivo patency of IBTA-based SD-TEVGs.

\subsection{Modification on SD-TEVGs}

As we have described above, many SD-TEVGs still fail, but chemical, biological and mechanical modifications may improve patency in the future. Indeed, a recent metaanalysis emphasized that such scaffold modifications improve patency, at least in large animals [122].

In surface modification, agents or bioactive molecules are attached or conjugated onto scaffolds by either physical or chemical treatments. This process reduces thrombogenicity and increases blood compatibility conferring general antithrombotic characteristics to the SD-TEVGs [131]. The most popular molecules used for surface modification embrace those possessing antithrombotic properties such as heparin, nitric oxide, tissue-type plasminogen activator (t-PA), thrombomodulin, prostacyclin, and their analogues [132]. In the retrieved large-animal studies, four studies exploited heparin coating of the TEVGs (Tables 4-7). As such, PCL graft was coated with heparin and tested without systemic antithrombotic treatment [60]. The coated PCL graft showed $100 \%$ patency after 28 days, which is similar to that observed in pure PCL tested with systemic antithrombotic treatment [45], suggesting that antithrombotic coating is effective. However, the former study was performed in a less challenging anastomosis fashion (ETE). Additionally, heparin has been coated onto decellularized autologous IBTA [73] and decellularized allogeneic canine carotid arteries combined with VEGF coating [75] and EC seeding [76], all showing favorable patencies (67-95\% after 60-180 days). However, since the combinations were tested in vivo under systemic antithrombotic treatment, the effectiveness of heparin coating alone is difficult to ascertain. Moreover, surface modifications tested that promote the adhesion and retention of ECs include coating with type I bovine collagen [70], fibronectin, alpha-2(VIII) collagen [72], POG7G3REDV peptide [49], P15 cell-binding peptide [47], and antihuman CD34 monoclonal antibodies [80]. Specific coating-like factor XIII has been explored to increase the crosslinking and strength of highly compacted fibrin TEVG [54], and semisynthetic heparan sulphate-like coating showed antiadhesive properties and seems to prevent neointimal formation [74]. Nevertheless, new approaches are continuously developed and include other molecules as well.

Biological modification refers to recellularization by in vitro repopulation of a scaffold with ECs and/or SMCs. This biomimics the native blood vessel structure and is therefore expected to improve SD-TEVG patency. It is well known that ECs contribute to thrombosis, as well as anticoagulant and fibrinolysis events under physiological conditions. Already, endothelialization has been shown to reduce SD-TEVG thrombogenicity and to improve outcomes [122,133]. Whereas ECs seem obligatory for in vivo SD-TEVG function, SMCs might be dispensable [122]. Among the studies included (Tables 4-7), and described above, ECs were usually isolated from veins [46,56,58], derived from fat [62], or differentiated from progenitor cells or stem cells from peripheral blood [54,57,78], or bone marrow [55,61]. SMC recellularization has only been applied in a few studies $[46,54-57,61,64,69]$, in which autologous SMCs were isolated from artery or vein or differentiated from progenitor cells in peripheral blood or bone marrow and seeded on the scaffold. However, the effectiveness of SMC recellularization alone is hard to define, since all these studies combined SMCs with ECs for recellularization. However, since arterial grafts in CABG patients has been reported to have a higher patency rate than venous grafts, SMCs might strengthen grafts and improve long-term patency [134]. The vigorous contribution from SMCs in modulating the graft is evidenced in a rat carotid model [135], where SMC seeding affected extracellular 
matrix composition in the intima and inhibited intimal hyperplasia [136], the latter being a major factor in medium- and long-term graft failure.

Moreover, to adapt the recellularized endothelial cells for a given arterial pressure and shear stress found in vivo, mechanical modification called preconditioning can be conducted using a peristaltic pump mimicking the palpating blood flow in vivo. Most of the retrieved large-animal studies that displayed excellent patency had seeded scaffolds with ECs $[46,51,54-58,61,62,64,65,69,72,76-78,81]$, which might be important for long-term SD-TEVG patency [137]. Alternatively, perfusion bioreactors have been designed to control both media flow and culture environment precisely in order to stimulate the recellularized cells mechanically and chemically [137]. However, most of the preconditioning studies performed in large animals were matching either the shear stress [51,64,65,69,76,77,81] or the pressure [46,78], whereas the graft in the in vivo situation has to withstand both of these two mechanical parameters in parallel to fulfill the physiological requirements. A perfusion bioreactor can imitate flow rate that is related to both shear stress and pressure. However, to achieve the physiological shear stress and pressure level in parallel in the bioreactor, it also requires that the contacting media have a viscosity similar to blood and the distal resistance of TEVG in culture is similar to that of the bypass graft in vivo. The desired viscosity can be achieved using culture medium supplemented with dextran and will result in endothelial properties suited for arteries [138] and the required resistance could be achieved by adding a specific length of tubing after the TEVG in the bioreactor loop system. Moreover, the bioreactors that includes rotational forces enable optimal perfusions throughout the 3-dimensional structure of bioengineered SD-TEVGs [139]. Thus, several modifications may be used to further improve overall patency of the resulting SD-TEVGs.

\subsection{In Situ SD-TEVGs Recellularization}

In vitro recellularization indeed seems to improve patency as reflected in the studies performed in large animals (Tables 4-7). However, the process is time consuming, which should be taken into consideration. This may also explain why the majority of studies still mainly investigate designs relying on in situ recellularization. There are three in situ recellularization mechanisms involved after implantation of SD-TEVGs: transanastomotic ingrowth, transmural capillarization, and fallout endothelialization [133]. Transanastomotic ingrowth occurs mainly in short TEVGs less than $2 \mathrm{~cm}$ with the involvement of EC proliferation from native vessel next to the proximal and distal anastomosis site [140-142]. However, this mechanism is insufficient when long grafts are implanted in patients, likely due to limited EC proliferation and senescence [141-143]. However, this knowledge is based entirely on synthetic TEVGs, while results await from nonsynthetic TEVGs, such as decellularized TEVGs. Transmural capillarization has been identified as capillaries that sprout from the granulation tissue in adventitia and grow through the graft towards the lumen $[144,145]$. These capillaries were found to be associated with the generation of endothelium in many synthetic grafts $[146,147]$. However, it is not easy to present the isolated effect of transmural capillarization, since it is not possible to exclude the role of another endothelialization mechanism referred as fallout endothelialization [148]. In fallout endothelialization, endothelial progenitor cells (EPCs) from the blood stream adhere to the graft through recognizing the protein absorbed on the surface of the graf, and here proliferate and differentiate into mature ECs $[149,150]$. Transmural capillarization is quite similar to angiogenesis during embryonic development, in which a capillary network is established after endothelial lumenization and fusion or extension of periphery blood islands made by endothelial cells [151]. Fallout endothelialization also seems to mimic developmental angiogenesis, since CD34+ progenitor cells are determined to contribute to both fallout endothelialization and embryological artery formation. During embryogenesis, angioblasts, and hematopoietic cells originate from a common precursor and arise almost at the same time in extraembryonic blood islands. In addition, CD34+ progenitor cells circulating in the blood have been shown to differentiate into endothelial cells [152]. Until now, the understanding of the biological mechanisms in recellularization arising after im- 
plantation of SD-TEVGs is still limited. More effort is needed to reveal these mechanisms in healthy as well as in diseased animals and individuals, so that the in vivo outcome of novel SD-TEVGs studies can benefit from the knowledge and further be translated into clinically relevant examinations.

\subsection{In Vivo SD-TEVG Graft Failure}

As described above, none of the current studies has developed an ideal SD-TEVG that do not, at some point, fail through different mechanisms. As a golden standard graft for $C A B G$, saphenous vein is used but fails due to thrombosis in the early phase, whereas intimal hyperplasia and atherosclerosis underline the failure in the intermediate and late phases, respectively [7]. SD-TEVG failure mechanisms during in vivo large-animal studies more or less equal to those found for saphenous vein $[153,154]$. Thrombus formation is primarily associated with low blood compatibility, compliance mismatch, exposure of a prothrombotic composition, lack of functional ECs, and poverty of proper antithrombotic molecules $[41,60,155,156]$. Intimal hyperplasia, on the other hand, causes SD-TEVG failure usually at medium term after implantation and reflects a range of factors (damaged ECs, inflammatory response against the graft material, deposition of micro thrombus, and change of hemodynamic forces). Such events might trigger the release of growth factors and further damage the ECM, which stimulates SMC proliferation and migration towards the intima layer and might also lead to deposition of new ECM, which finally narrows the SD-TEVG lumen [153,157]. SMCs involved in intimal hyperplasia are suggested to originate from (1) SMCs that switch from a "contractile" to a "synthetic" SMCs, or (2) circulating precursors (e.g., mesenchymal stem cells, bone marrow derived progenitor cells, and monocytes), or (3) adventitia derived fibroblasts [157-159]. Through interaction with glycosaminoglycan in the ECM, intimal hyperplasia further accelerates lipoprotein retention, which develops into atherosclerotic plaques [160,161]. Being highly susceptible to thrombus, atherosclerotic plaques thus increase the risk of graft occlusion and is a major reason for SD-TEVG failure. In contrast, graft rupture or aneurysm is less frequent, since they can be avoided by mechanical testing before in vivo evaluation in animal models. However, mechanical strength can still be weakened after implantation due to rapid scaffold degradation or insufficient ECM deposition by host cells. In the large-animal studies, mechanical failures (Tables 4-7) such as rupture [54,79], prosthetic kink [68,81], delamination [74], or dilation [57] have been reported, however they are not observed in the rest of the studies. Thrombus remains the dominant failure mechanism observed until now and may be reduced through application of systemic antithrombotic treatment $[44,45]$. The second often seen failure mechanism is intimal hyperplasia (Tables 4-7) $[50,71,73,74]$, occurring at the anastomosis site. Regarding allogeneic native ECM-based SD-TEVGs, some studies report thrombus as failure $[55,61]$, whereas our sheep study reports that the thrombus was absent but instead failure was due to distal anastomotic stenosis resulting from intimal hyperplasia in the allogeneic dSCA [50]. Such stenosis however leads to thrombus in the end because of blood stasis according to "Virchow's triad". It would be desirable if the existences of intimal hyperplasia could be clearly distinguished from thrombus occurring in the intermediate phase $[75,76]$. In contrast to thrombus and intimal hyperplasia, atherosclerosis has not been reported as a reason for graft failure in large animals yet (Tables 4-7). This is most likely explained by the fact that atherosclerosis only becomes relevant upon long-term patency, which has not yet been achieved for SD-TEVGs in large animals.

Thus, in general only a few causes underlie SD-TEVG graft failure, which may seem simple in nature of prevention. However, in reality these challenges have persisted after decades of research and still needs to be solved to achieve clinical success. Whereas proper mechanical strength seems implemented with mechanical failures like ruptures being seldomly reported in large-animal studies, further improvement of biocompatibility to prevent thrombus and intimal hyperplasia is required. 


\section{Conclusions and Perspectives}

Until now, a large range of materials have been tested as SD-TEVGs, including synthetic polymers, natural molecular, or a combination of these, as xenogeneic or allogeneic grafts, with or without recellularization of autologous cells. However, studies with high clinical relevance, such as those performed in large-animal models, are still limited in numbers, and most of the studies provide little success: either by limited patency of the grafts or very short observation time. Long-term ( $>12$ months) patency of the grafts is generally not observed except for one study reporting PEtU-PDMS-based SD-TEVGs manifesting $100 \%$ patency even after 2 years with application of both end-to-side anastomosis and antithrombotic treatment [53]. Despite being published already back in 2010, no further clinical study has been reported for these PEtU-PDMS-based SD-TEVGs.

Although, we have tried to categorize large-animal studies testing SD-TEVGs for comparison, direct comparisons are indeed difficult, since SD-TEVG and study designs vary a lot. Two parameters important for clinical translation relate to the anastomotic fashion (ETS and ETE) and the systemic antithrombotic treatment. ETS anastomosis is used in CABG and other bypass surgeries, while ETE anastomosis is much less used in clinical settings. As compared to ETE anastomosis, ETS anastomosis represents a more challenging situation, since complicated hemodynamic characteristics provoke adverse biological responses from the surrounding tissue and blood components. This in turn might influence intimal hyperplasia and thrombus formation, the two most common graft failure mechanisms. For instance, Anderson et al., have reported $66 \%$ patency when using ETS anastomosis as compared to $100 \%$ patency for ETE anastomosis [162]. Considering that 25 out of 32 large-animal studies performed ETE surgery when testing SD-TEVGs as arterial bypass grafts, it seems reasonable to speculate that reported SD-TEVG patency in general may be exaggerated in the field, and that ETS should be considered for future studies.

Likewise, and as mentioned several times above, systemic application of antithrombotic therapy is a commonly used treatment after bypass surgery in clinical practice due to its substantial improvement on patency [163]. This is also clearly supported in the identified literature of large-animal studies testing SD-TEVGs, and $66 \%$ (26 out of 39) of large-animal studies have already adopted antithrombotic treatment (Tables 4 and 6). In particular, direct comparisons have shown that pure PCL SD-TEVGs exhibit a 100 versus $0 \%$ patency upon antithrombotic treatment after 4 weeks [45]. Likewise, Weber et al. reported a $67 \%$ patency of a bacterial cellulose tube on day 270 with systemic antithrombotic treatment, while bacterial cellulose tube without systemic antithrombotic treatment displayed failure already 56 days after implantation [44]. Thus, even though the lack of antithrombotic treatment may seem ideal, the field may overlook important candidate SD-TEVGs, if systemic antithrombotic treatment is avoided. Moreover, it should be noted that occlusions still occur under systemic antithrombotic treatment, especially when the observation period is extended $[62,68,74]$. Long-term follow up seems therefore required to reveal the effect of experimental factors on graft patency when systemic antithrombotic treatment is involved.

Besides the testing model-related parameters, graft material and modifications are determinants for graft outcome in large-animal models. Graft rupture due to weak strength is still an observed failure mechanism, especially in natural polymer- and cell-secreted ECM-based SD-TEVGs, whereas synthetic SD-TEVGs can be fabricated with adjustable compliance and strength. However, natural SD-TEVGs, especially nonxenogeneic ones, display higher biocompatibility than synthetic SD-TEVGs, and they are therefore more protected from thrombus, which is the dominant type of graft failure in large-animal models until now. In native ECM-based SD-TEVGs and hybrid SD-TEVGs, xenogeneic originated molecular or matrix seems to harm the graft outcome. Thus, in vitro developed hybrid SD-TEVGs using allogeneic banked human cells or isolated autologous stem cells would be an optimal choice to avoid xenogeneic material. Beside this, it seems that allogeneic native ECM-based SD-TEVGs and IBTA-based SD-TEVGs of autologous or 
allogeneic origins may be adequate choices as well. However, allogeneic human grafts should be obtained from either cadaveric donor or from waste sources such as dHUA SD-TEVGs. Tissue of origin and unknown underlying diseases may affect SD-TEVG quality, and be uncontrollable factors increasing the cost to SD-TEVG fabrication in a large scale. Besides the choice of graft origin, modifications such as autologous ECs seeding, and preconditioning are showing significant effect in maintaining graft patency and are therefore strongly recommended prior to implantation. To further manipulate the SDTEVGs and ameliorate the in vivo outcome, solutions to issues such as stem cell sources for repopulating the media compartment and substituting the ECs would also be of great value. Moreover, the association between the ECM ultrastructure and vessel cell retention is important as are signaling between host cells and graft components and the continued remodeling of grafts after implantation.

Thus, the field of SD-TEVG is continuously evolving, but even after decades of research, several challenges remain and require further investigation before the concept of SD-TEVG can be translated into standard care in humans.

Author Contributions: Conceptualization, S.F. and D.C.A.; methodology, S.F. and D.C.A.; formal analysis, S.F.; investigation, S.F., D.G.E. and D.C.A.; writing-original draft preparation, S.F.; writingreview and editing, S.F., D.G.E. and D.C.A.; visualization, S.F.; supervision, D.G.E. and D.C.A.; project administration, S.F. and D.C.A.; funding acquisition, S.F. and D.C.A. All authors have read and agreed to the published version of the manuscript.

Funding: This research was funded by the research finance from Department of Clinical Biochemistry and Pharmacology (OUH); strategic grant from Odense University Hospital; Karen Elise Jensen; University of Southern Denmark, the Innovation Fund Denmark (\#7051-00001A) and S.C. Van Fonden (\#1530).

Institutional Review Board Statement: Not applicable.

Informed Consent Statement: Not applicable.

Data Availability Statement: No new data were created or analyzed in this study. Data sharing is not applicable to this article.

Conflicts of Interest: The authors declare no conflict of interest.

\section{References}

1. Mendis, S.; Puska, P.; Norrving, B.; World Health Organization. Global Atlas on Cardiovascular Disease Prevention and Control; Mendis, S., Puska, P., Norrving, B., Eds.; World Health Organization: Geneva, Switzerland, 2011.

2. Eurostat. Causes of Death Statistics. Available online: https://ec.europa.eu/eurostat/databrowser/view/hlth_cd_asdr2/default/ table?lang=en (accessed on 19 January 2021).

3. Carrel, T.; Winkler, B. Current Trends in Selection of Conduits for Coronary Artery Bypass Grafting. Gen. Thorac. Cardiovasc. Surg. 2017, 65, 549-556. [CrossRef]

4. Martinez-Gonzalez, B.; Reyes-Hernandez, C.G.; Quiroga-Garza, A.; Rodriguez-Rodriguez, V.E.; Esparza-Hernandez, C.N.; Elizondo-Omana, R.E.; Guzman-Lopez, S. Conduits Used in Coronary Artery Bypass Grafting: A Review of Morphological Studies. Ann. Thorac. Cardiovasc. Surg. 2017, 23, 55-65. [CrossRef]

5. Goldman, S.; Zadina, K.; Moritz, T.; Ovitt, T.; Sethi, G.; Copeland, J.G.; Thottapurathu, L.; Krasnicka, B.; Ellis, N.; Anderson, R.J.; et al. Long-Term Patency of Saphenous Vein and Left Internal Mammary Artery Grafts after Coronary Artery Bypass Surgery: Results from a Department of Veterans Affairs Cooperative Study. J. Am. Coll. Cardiol. 2004, 44, 2149-2156. [CrossRef] [PubMed]

6. Tinica, G.; Chistol, R.O.; Enache, M.; Constantin, M.M.L.; Ciocoiu, M.; Furnica, C. Long-Term Graft Patency after Coronary Artery Bypass Grafting: Effects of Morphological and Pathophysiological Factors. Anatol. J. Cardiol. 2018, 20, 275-282. [CrossRef]

7. Caliskan, E.; de Souza, D.R.; Boning, A.; Liakopoulos, O.J.; Choi, Y.H.; Pepper, J.; Gibson, C.M.; Perrault, L.P.; Wolf, R.K.; Kim, K.B.; et al. Saphenous Vein Grafts in Contemporary Coronary Artery Bypass Graft Surgery. Nat. Rev. Cardiol. 2019, 17, 155-169. [CrossRef] [PubMed]

8. Ambler, K.G.; Twine, C.P. Graft Type for Femoro-Popliteal Bypass Surgery. Cochrane Database Syst. Rev. 2010. [CrossRef] [PubMed]

9. Arvela, E.; Venermo, M.; Soderstrom, M.; Alback, A.; Lepantalo, M. Outcome of Infrainguinal Single-Segment Great Saphenous Vein Bypass for Critical Limb Ischemia Is Superior to Alternative Autologous Vein Bypass, Especially in Patients with High Operative Risk. Ann. Vasc. Surg. 2012, 26, 396-403. [CrossRef] [PubMed]

10. Handa, R.; Sharma, S. Vascular Graft Failure of Leg Arterial Bypasses-A Review. J. Hypertens. Cardiol. 2014, 1, 17-21. [CrossRef] 
11. Spinelli, F.; Benedetto, F.; Passari, G.; la Spada, M.; Carella, G.; Stilo, F.; de Caridi, G.; Lentini, S. Bypass Surgery for the Treatment of Upper Limb Chronic Ischaemia. Eur. J. Vasc. Endovasc. Surg. 2010, 39, 165-170. [CrossRef]

12. Chandra, P.; Atala, A. Engineering Blood Vessels and Vascularized Tissues: Technology Trends and Potential Clinical Applications. Clin. Sci. 2019, 133, 1115-1135. [CrossRef]

13. Hoenig, R.M.; Campbell, G.R.; Rolfe, B.E.; Campbell, J.H. Tissue-Engineered Blood Vessels: Alternative to Autologous Grafts? Arterioscler. Thromb. Vasc. Biol. 2005, 25, 1128-1134. [CrossRef]

14. Lorbeer, R.; Grotz, A.; Dorr, M.; Volzke, H.; Lieb, W.; Kuhn, J.P.; Mensel, B. Reference Values of Vessel Diameters, Stenosis Prevalence, and Arterial Variations of the Lower Limb Arteries in a Male Population Sample Using Contrast-Enhanced Mr Angiography. PLoS ONE 2018, 13, e0197559. [CrossRef] [PubMed]

15. Vartanian, S.M.; Conte, M.S. Surgical Intervention for Peripheral Arterial Disease. Circ. Res. 2015, 116, 614-628. [CrossRef] [PubMed]

16. Unlu, Y.; Keles, P.; Keles, S.; Yesilyurt, H.; Kocak, H.; Diyarbakirli, S. An Evaluation of Histomorphometric Properties of Coronary Arteries, Saphenous Vein, and Various Arterial Conduits for Coronary Artery Bypass Grafting. Surg. Today 2003, 33, 725-730. [CrossRef]

17. Sauvage, R.L.; Schloemer, R.; Wood, S.J.; Logan, G. Successful Interposition Synthetic Graft between Aorta and Right Coronary Artery. Angiographic Follow-up to Sixteen Months. J. Thorac. Cardiovasc. Surg. 1976, 72, 418-421. [CrossRef]

18. Hallman, L.G.; Cooley, D.A.; McNamara, D.G.; Latson, J.R. Single Left Coronary Artery with Fistula to Right Ventricle: Reconstruction of Two-Coronary System with Dacron Graft. Circulation 1965, 32, 293-297. [CrossRef]

19. Desai, M.; Seifalian, A.M.; Hamilton, G. Role of Prosthetic Conduits in Coronary Artery Bypass Grafting. Eur. J. Cardiothorac. Surg. 2011, 40, 394-398. [CrossRef]

20. Silver, M.G.; Katske, G.E.; Stutzman, F.L.; Wood, N.E. Umbilical Vein for Aortocoronary Bypass. Angiology 1982, $33,450-453$. [CrossRef]

21. Laub, W.G.; Muralidharan, S.; Clancy, R.; Eldredge, W.J.; Chen, C.; Adkins, M.S.; Fernandez, J.; Anderson, W.A.; McGrath, L.B. Cryopreserved Allograft Veins as Alternative Coronary Artery Bypass Conduits: Early Phase Results. Ann. Thorac. Surg. 1992, 54, 826-831. [CrossRef]

22. Mitchell, M.I.; Essop, A.R.; Scott, P.J.; Martin, P.G.; Gupta, N.K.; Saunders, N.R.; Nair, R.U.; Williams, G.J. Bovine Internal Mammary Artery as a Conduit for Coronary Revascularization: Long-Term Results. Ann. Thorac. Surg. 1993, 55, 120-122. [CrossRef]

23. Reddy, L.S.; Pillai, J.; Mitchell, L.; Naik, S.; Dark, J.; Hasan, A.; Ledingham, S.; Clark, S.C. First Report of No-React Bovine Internal Mammary Artery Performance and Patency. Heart Surg. Forum 2004, 7, E446-E449. [CrossRef] [PubMed]

24. Englberger, L.; Noti, J.; Immer, F.F.; Stalder, M.; Eckstein, F.S.; Carrel, T.P. The Shelhigh No-React Bovine Internal Mammary Artery: A Questionable Alternative Conduit in Coronary Bypass Surgery? Eur. J. Cardiothorac. Surg. 2008, 33, 222-224. [CrossRef] [PubMed]

25. Laube, R.H.; Duwe, J.; Rutsch, W.; Konertz, W. Clinical Experience with Autologous Endothelial Cell-Seeded Polytetrafluoroethylene Coronary Artery Bypass Grafts. J. Thorac. Cardiovasc. Surg. 2000, 120, 134-141. [CrossRef] [PubMed]

26. Lamm, P.; Juchem, G.; Milz, S.; Schuffenhauer, M.; Reichart, B. Autologous Endothelialized Vein Allograft: A Solution in the Search for Small-Caliber Grafts in Coronary Artery Bypass Graft Operations. Circulation 2001, 104, I108-I114. [CrossRef]

27. Herrmann, M.F.E.; Lamm, P.; Wellmann, P.; Milz, S.; Hagl, C.; Juchem, G. Autologous Endothelialized Vein Allografts in Coronary Artery Bypass Surgery-Long Term Results. Biomaterials 2019, 212, 87-97. [CrossRef]

28. Almasri, J.; Adusumalli, J.; Asi, N.; Lakis, S.; Alsawas, M.; Prokop, L.J.; Bradbury, A.; Kolh, P.; Conte, M.S.; Murad, M.H. A Systematic Review and Meta-Analysis of Revascularization Outcomes of Infrainguinal Chronic Limb-Threatening Ischemia. $J$. Vasc. Surg. 2018, 68, 624-633. [CrossRef]

29. Lindsey, P.; Echeverria, A.; Cheung, M.; Kfoury, E.; Bechara, C.F.; Lin, P.H. Lower Extremity Bypass Using Bovine Carotid Artery Graft (Artegraft): An Analysis of 124 Cases with Long-Term Results. World J. Surg. 2018, 42, 295-301. [CrossRef] [PubMed]

30. Williams, S.K.; Morris, M.E.; Kosnik, P.E.; Lye, K.D.; Gentzkow, G.D.; Ross, C.B.; Dwevidi, A.J.; Kleinert, L.B. Point-of-Care Adipose-Derived Stromal Vascular Fraction Cell Isolation and Expanded Polytetrafluoroethylene Graft Sodding. Tissue Eng. Part C Methods 2017, 23, 497-504. [CrossRef]

31. Kennealey, P.T.; Elias, N.; Hertl, M.; Ko, D.S.; Saidi, R.F.; Markmann, J.F.; Smoot, E.E.; Schoenfeld, D.A.; Kawai, T. A Prospective, Randomized Comparison of Bovine Carotid Artery and Expanded Polytetrafluoroethylene for Permanent Hemodialysis Vascular Access. J. Vasc. Surg. 2011, 53, 1640-1648. [CrossRef] [PubMed]

32. Harlander-Locke, M.; Jimenez, J.C.; Lawrence, P.F.; Gelabert, H.A.; Derubertis, B.G.; Rigberg, D.A.; Farley, S.M. Bovine Carotid Artery (Artegraft) as a Hemodialysis Access Conduit in Patients Who Are Poor Candidates for Native Arteriovenous Fistulae. Vasc. Endovasc. Surg. 2014, 48, 497-502. [CrossRef] [PubMed]

33. Wystrychowski, W.; McAllister, T.N.; Zagalski, K.; Dusserre, N.; Cierpka, L.; L'Heureux, N. First Human Use of an Allogeneic Tissue-Engineered Vascular Graft for Hemodialysis Access. J. Vasc. Surg. 2014, 60, 1353-1357. [CrossRef] [PubMed]

34. Lawson, J.H.; Glickman, M.H.; Ilzecki, M.; Jakimowicz, T.; Jaroszynski, A.; Peden, E.K.; Pilgrim, A.J.; Prichard, H.L.; Guziewicz, M.; Przywara, S.; et al. Bioengineered Human Acellular Vessels for Dialysis Access in Patients with End-Stage Renal Disease: Two Phase 2 Single-Arm Trials. Lancet 2016, 387, 2026-2034. [CrossRef] 
35. L'Heureux, N.; McAllister, T.N.; de la Fuente, L.M. Tissue-Engineered Blood Vessel for Adult Arterial Revascularization. N. Engl. J. Med. 2007, 357, 1451-1453. [CrossRef] [PubMed]

36. McAllister, T.N.; Maruszewski, M.; Garrido, S.A.; Wystrychowski, W.; Dusserre, N.; Marini, A.; Zagalski, K.; Fiorillo, A.; Avila, H.; Manglano, X.; et al. Effectiveness of Haemodialysis Access with an Autologous Tissue-Engineered Vascular Graft: A Multicentre Cohort Study. Lancet 2009, 373, 1440-1446. [CrossRef]

37. Wystrychowski, W.; Cierpka, L.; Zagalski, K.; Garrido, S.; Dusserre, N.; Radochonski, S.; McAllister, T.N.; L’Heureux, N. Case Study: First Implantation of a Frozen, Devitalized Tissue-Engineered Vascular Graft for Urgent Hemodialysis Access. J. Vasc. Access 2011, 12, 67-70. [CrossRef]

38. Goodman, S.L. Sheep, Pig, and Human Platelet-Material Interactions with Model Cardiovascular Biomaterials. J. Biomed. Mater. Res. 1999, 45, 240-250. [CrossRef]

39. Pichler, L. Parameters of Coagulation and Fibrinolysis in Different Animal Species-A Literature Based Comparison. Wien Tierärtzl. Mschr. 2008, 95, 282.

40. Gui, L.; Muto, A.; Chan, S.A.; Breuer, C.K.; Niklason, L.E. Development of Decellularized Human Umbilical Arteries as Small-Diameter Vascular Grafts. Tissue Eng. Part A 2009, 15, 2665-2676. [CrossRef]

41. Zheng, W.; Wang, Z.; Song, L.; Zhao, Q.; Zhang, J.; Li, D.; Wang, S.; Han, J.; Zheng, X.L.; Yang, Z.; et al. Endothelialization and Patency of Rgd-Functionalized Vascular Grafts in a Rabbit Carotid Artery Model. Biomaterials 2012, 33, 2880-2891. [CrossRef] [PubMed]

42. Thomas, L.V.; Lekshmi, V.; Nair, P.D. Tissue Engineered Vascular Grafts—Preclinical Aspects. Int. J. Cardiol. 2013, 167, 1091-1100. [CrossRef]

43. Fukunishi, T.; Best, C.A.; Sugiura, T.; Shoji, T.; Yi, T.; Udelsman, B.; Ohst, D.; Ong, C.S.; Zhang, H.; Shinoka, T.; et al. TissueEngineered Small Diameter Arterial Vascular Grafts from Cell-Free Nanofiber Pcl/Chitosan Scaffolds in a Sheep Model. PLoS ONE 2016, 11, e0158555. [CrossRef]

44. Weber, C.; Reinhardt, S.; Eghbalzadeh, K.; Wacker, M.; Guschlbauer, M.; Maul, A.; Sterner-Kock, A.; Wahlers, T.; Wippermann, J.; Scherner, M. Patency and in Vivo Compatibility of Bacterial Nanocellulose Grafts as Small-Diameter Vascular Substitute. J. Vasc. Surg. 2018, 68, 177S-187S. [CrossRef]

45. Fang, S.; Ahlmann, A.H.; Langhorn, L.; Hussein, K.; Sørensen, J.A.; Guan, X.W.; Sheikh, S.P.; Riber, L.P.; Andersen, D.C. Small Diameter Polycaprolactone Vascular Grafts Are Patent in Sheep Carotid Bypass but Require Anti-Thrombotic Therapy. Regen. Med.. In Press.

46. Dahan, N.; Sarig, U.; Bronshtein, T.; Baruch, L.; Karram, T.; Hoffman, A.; Machluf, M. Dynamic Autologous Reendothelialization of Small-Caliber Arterial Extracellular Matrix: A Preclinical Large Animal Study. Tissue Eng. Part A 2017, 23, 69-79. [CrossRef]

47. Li, C.; Hill, A.; Imran, M. In Vitro and in Vivo Studies of Eptfe Vascular Grafts Treated with P15 Peptide. J. Biomater. Sci. Polym. Ed. 2005, 16, 875-891. [CrossRef]

48. Langer, R.; Vacanti, J.P. Tissue Engineering. Science 1993, 260, 920-926. [CrossRef] [PubMed]

49. Mahara, A.; Somekawa, S.; Kobayashi, N.; Hirano, Y.; Kimura, Y.; Fujisato, T.; Yamaoka, T. Tissue-Engineered Acellular Small Diameter Long-Bypass Grafts with Neointima-Inducing Activity. Biomaterials 2015, 58, 54-62. [CrossRef] [PubMed]

50. Fang, S.; Riber, S.S.; Hussein, K.; Ahlmann, A.H.; Harvald, E.B.; Khan, F.; Beck, H.C.; Weile, L.K.K.; Sorensen, J.A.; Sheikh, S.P.; et al. Decellularized Human Umbilical Artery: Biocompatibility and in Vivo Functionality in Sheep Carotid Bypass Model. Mater. Sci. Eng. C Mater. Biol. Appl. 2020, 112, 110955. [CrossRef] [PubMed]

51. Dahl, S.L.; Kypson, A.P.; Lawson, J.H.; Blum, J.L.; Strader, J.T.; Li, Y.; Manson, R.J.; Tente, W.E.; DiBernardo, L.; Hensley, M.T.; et al. Readily Available Tissue-Engineered Vascular Grafts. Sci. Transl. Med. 2011, 3, 68ra9. [CrossRef]

52. Nakayama, Y.; Furukoshi, M.; Terazawa, T.; Iwai, R. Development of Long in Vivo Tissue-Engineered Biotube Vascular Grafts. Biomaterials 2018, 185, 232-239. [CrossRef]

53. Soldani, G.; Losi, P.; Bernabei, M.; Burchielli, S.; Chiappino, D.; Kull, S.; Briganti, E.; Spiller, D. Long Term Performance of Small-Diameter Vascular Grafts Made of a Poly(Ether)Urethane-Polydimethylsiloxane Semi-Interpenetrating Polymeric Network. Biomaterials 2010, 31, 2592-2605. [CrossRef]

54. Aper, T.; Wilhelmi, M.; Gebhardt, C.; Hoeffler, K.; Benecke, N.; Hilfiker, A.; Haverich, A. Novel Method for the Generation of Tissue-Engineered Vascular Grafts Based on a Highly Compacted Fibrin Matrix. Acta Biomater. 2016, 29, 21-32. [CrossRef] [PubMed]

55. Cho, S.W.; Lim, S.H.; Kim, I.K.; Hong, Y.S.; Kim, S.S.; Yoo, K.J.; Park, H.Y.; Jang, Y.; Chang, B.C.; Choi, C.Y.; et al. Kim, B.S. Small-Diameter Blood Vessels Engineered with Bone Marrow-Derived Cells. Ann. Surg. 2005, 241, 506-515. [CrossRef]

56. He, H.; Matsuda, T. Arterial Replacement with Compliant Hierarchic Hybrid Vascular Graft: Biomechanical Adaptation and Failure. Tissue Eng. 2002, 8, 213-224. [CrossRef] [PubMed]

57. He, H.; Shirota, T.; Yasui, H.; Matsuda, T. Canine Endothelial Progenitor Cell-Lined Hybrid Vascular Graft with Nonthrombogenic Potential. J. Thorac. Cardiovasc. Surg. 2003, 126, 455-464. [CrossRef]

58. Narita, Y.; Kagami, H.; Matsunuma, H.; Murase, Y.; Ueda, M.; Ueda, Y. Decellularized Ureter for Tissue-Engineered Small-Caliber Vascular Graft. J. Artif. Organs 2008, 11, 91-99. [CrossRef]

59. Scherner, M.; Reutter, S.; Klemm, D.; Sterner-Kock, A.; Guschlbauer, M.; Richter, T.; Langebartels, G.; Madershahian, N.; Wahlers, T.; Wippermann, J. In Vivo Application of Tissue-Engineered Blood Vessels of Bacterial Cellulose as Small Arterial Substitutes: Proof of Concept? J. Surg. Res. 2014, 189, 340-347. [CrossRef] [PubMed] 
60. Ye, L.; Wu, X.; Duan, H.Y.; Geng, X.; Chen, B.; Gu, Y.Q.; Zhang, A.Y.; Zhang, J.; Feng, Z.G. The in Vitro and in Vivo Biocompatibility Evaluation of Heparin-Poly(Epsilon-Caprolactone) Conjugate for Vascular Tissue Engineering Scaffolds. J. Biomed. Mater. Res. A 2012, 100, 3251-3258. [CrossRef] [PubMed]

61. Zhao, Y.; Zhang, S.; Zhou, J.; Wang, J.; Zhen, M.; Liu, Y.; Chen, J.; Qi, Z. The Development of a Tissue-Engineered Artery Using Decellularized Scaffold and Autologous Ovine Mesenchymal Stem Cells. Biomaterials 2010, 31, 296-307. [CrossRef]

62. Arts, C.H.; Hedeman Joosten, P.P.; Blankensteijn, J.D.; Staal, F.J.; Ng, P.Y.; Heijnen-Snyder, G.J.; Sixma, J.J.; Verhagen, H.J.; de Groot, P.G.; Eikelboom, B.C. Contaminants from the Transplant Contribute to Intimal Hyperplasia Associated with Microvascular Endothelial Cell Seeding. Eur. J. Vasc. Endovasc. Surg. 2002, 23, 29-38. [CrossRef]

63. Chue, W.L.; Campbell, G.R.; Caplice, N.; Muhammed, A.; Berry, C.L.; Thomas, A.C.; Bennett, M.B.; Campbell, J.H. Dog Peritoneal and Pleural Cavities as Bioreactors to Grow Autologous Vascular Grafts. J. Vasc. Surg. 2004, 39, 859-867. [CrossRef]

64. Ju, Y.M.; Ahn, H.; Arenas-Herrera, J.; Kim, C.; Abolbashari, M.; Atala, A.; Yoo, J.J.; Lee, S.J. Electrospun Vascular Scaffold for Cellularized Small Diameter Blood Vessels: A Preclinical Large Animal Study. Acta Biomater. 2017, 59, 58-67. [CrossRef] [PubMed]

65. Kaushal, S.; Amiel, G.E.; Guleserian, K.J.; Shapira, O.M.; Perry, T.; Sutherland, F.W.; Rabkin, E.; Moran, A.M.; Schoen, F.J.; Atala, A.; et al. Functional Small-Diameter Neovessels Created Using Endothelial Progenitor Cells Expanded Ex Vivo. Nat. Med. 2001, 7, 1035-1040. [CrossRef] [PubMed]

66. L'Heureux, N.; Paquet, S.; Labbe, R.; Germain, L.; Auger, F.A. A Completely Biological Tissue-Engineered Human Blood Vessel. FASEB J. 1998, 12, 47-56.

67. Ma, X.; He, Z.; Li, L.; Liu, G.; Li, Q.; Yang, D.; Zhang, Y.; Li, N. Development and in Vivo Validation of Tissue-Engineered, Small-Diameter Vascular Grafts from Decellularized Aortae of Fetal Pigs and Canine Vascular Endothelial Cells. J. Cardiothorac. Surg. 2017, 12, 101. [CrossRef]

68. Mrowczynski, W.; Mugnai, D.; de Valence, S.; Tille, J.C.; Khabiri, E.; Cikirikcioglu, M.; Moller, M.; Walpoth, B.H. Porcine Carotid Artery Replacement with Biodegradable Electrospun Poly-E-Caprolactone Vascular Prosthesis. J. Vasc. Surg. 2014, 59, $210-219$. [CrossRef]

69. Neff, L.P.; Tillman, B.W.; Yazdani, S.K.; Machingal, M.A.; Yoo, J.J.; Soker, S.; Bernish, B.W.; Geary, R.L.; Christ, G.J. Vascular Smooth Muscle Enhances Functionality of Tissue-Engineered Blood Vessels in Vivo. J. Vasc. Surg. 2011, 53, 426-434. [CrossRef]

70. Nemcova, S.; Noel, A.A.; Jost, C.J.; Gloviczki, P.; Miller, V.M.; Brockbank, K.G. Evaluation of a Xenogeneic Acellular Collagen Matrix as a Small-Diameter Vascular Graft in Dogs-Preliminary Observations. J. Investig. Surg. 2001, 14, 321-330. [CrossRef]

71. Rothuizen, T.C.; Damanik, F.F.R.; Lavrijsen, T.; Visser, M.J.T.; Hamming, J.F.; Lalai, R.A.; Duijs, J.; van Zonneveld, A.J.; Hoefer, I.E.; van Blitterswijk, C.A.; et al. Development and Evaluation of in Vivo Tissue Engineered Blood Vessels in a Porcine Model. Biomaterials 2016, 75, 82-90. [CrossRef]

72. Turner, N.J.; Murphy, M.O.; Kielty, C.M.; Shuttleworth, C.A.; Black, R.A.; Humphries, M.J.; Walker, M.G.; Canfield, A.E. Alpha2(Viii) Collagen Substrata Enhance Endothelial Cell Retention under Acute Shear Stress Flow Via an Alpha2beta1 IntegrinDependent Mechanism: An in Vitro and in Vivo Study. Circulation 2006, 114, 820-829. [CrossRef] [PubMed]

73. Wang, T.; Dong, N.; Yan, H.; Wong, S.Y.; Zhao, W.; Xu, K.; Wang, D.; Li, S.; Qiu, X. Regeneration of a Neoartery through a Completely Autologous Acellular Conduit in a Minipig Model: A Pilot Study. J. Transl. Med. 2019, 17, 24. [CrossRef] [PubMed]

74. Wulff, B.; Stahlhoff, S.; Vonthein, R.; Schmidt, A.; Sigler, M.; Torsello, G.B.; Herten, M. Biomimetic Heparan Sulfate-Like Coated Eptfe Grafts Reduce in-Graft Neointimal Hyperplasia in Ovine Carotids. Ann. Vasc. Surg. 2017, 40, 274-284. [CrossRef] [PubMed]

75. Zhou, M.; Liu, Z.; Wei, Z.; Liu, C.; Qiao, T.; Ran, F.; Bai, Y.; Jiang, X.; Ding, Y. Development and Validation of Small-Diameter Vascular Tissue from a Decellularized Scaffold Coated with Heparin and Vascular Endothelial Growth Factor. Artif. Organs 2009, 33, 230-239. [CrossRef]

76. Zhou, M.; Liu, Z.; Liu, C.; Jiang, X.; Wei, Z.; Qiao, W.; Ran, F.; Wang, W.; Qiao, T.; Liu, C. Tissue Engineering of Small-Diameter Vascular Grafts by Endothelial Progenitor Cells Seeding Heparin-Coated Decellularized Scaffolds. J. Biomed. Mater. Res. B Appl. Biomater. 2012, 100, 111-120. [CrossRef]

77. Zhou, M.; Qiao, W.; Liu, Z.; Shang, T.; Qiao, T.; Mao, C.; Liu, C. Development and in Vivo Evaluation of Small-Diameter Vascular Grafts Engineered by Outgrowth Endothelial Cells and Electrospun Chitosan/Poly(Epsilon-Caprolactone) Nanofibrous Scaffolds. Tissue Eng. Part A 2014, 20, 79-91. [CrossRef]

78. Koenneker, S.; Teebken, O.E.; Bonehie, M.; Pflaum, M.; Jockenhoevel, S.; Haverich, A.; Wilhelmi, M.H. A Biological Alternative to Alloplastic Grafts in Dialysis Therapy: Evaluation of an Autologised Bioartificial Haemodialysis Shunt Vessel in a Sheep Model. Eur. J. Vasc. Endovasc. Surg. 2010, 40, 810-816. [CrossRef] [PubMed]

79. Syedain, Z.H.; Graham, M.L.; Dunn, T.B.; O’Brien, T.; Johnson, S.L.; Schumacher, R.J.; Tranquillo, R.T. A Completely Biological Off-the-Shelf Arteriovenous Graft That Recellularizes in Baboons. Sci. Transl. Med. 2017, 9. [CrossRef]

80. Rotmans, J.I.; Heyligers, J.M.; Verhagen, H.J.; Velema, E.; Nagtegaal, M.M.; de Kleijn, D.P.; de Groot, F.G.; Stroes, E.S.; Pasterkamp, G. In Vivo Cell Seeding with Anti-Cd34 Antibodies Successfully Accelerates Endothelialization but Stimulates Intimal Hyperplasia in Porcine Arteriovenous Expanded Polytetrafluoroethylene Grafts. Circulation 2005, 112, 12-18. [CrossRef]

81. Tillman, B.W.; Yazdani, S.K.; Neff, L.P.; Corriere, M.A.; Christ, G.J.; Soker, S.; Atala, A.; Geary, R.L.; Yoo, J.J. Bioengineered Vascular Access Maintains Structural Integrity in Response to Arteriovenous Flow and Repeated Needle Puncture. J. Vasc. Surg. 2012, 56, 783-793. [CrossRef] 
82. Ong, C.S.; Fukunishi, T.; Liu, R.H.; Nelson, K.; Zhang, H.; Wieczorek, E.; Palmieri, M.; Ueyama, Y.; Ferris, E.; Geist, G.E.; et al. Bilateral Arteriovenous Shunts as a Method for Evaluating Tissue-Engineered Vascular Grafts in Large Animal Models. Tissue Eng. Part C Methods 2017, 23, 728-735. [CrossRef]

83. Furukoshi, M.; Tatsumi, E.; Nakayama, Y. Application of in-Body Tissue Architecture-Induced Biotube Vascular Grafts for Vascular Access: Proof of Concept in a Beagle Dog Model. J. Vasc. Access 2019, 21, 314-321. [CrossRef] [PubMed]

84. Seifalian, A.M.; Salacinski, H.J.; Tiwari, A.; Edwards, A.; Bowald, S.; Hamilton, G. In Vivo Biostability of a Poly(CarbonateUrea)Urethane Graft. Biomaterials 2003, 24, 2549-2557. [CrossRef]

85. Zeugolis, D.I.; Khew, S.T.; Yew, E.S.; Ekaputra, A.K.; Tong, Y.W.; Yung, L.Y.; Hutmacher, D.W.; Sheppard, C.; Raghunath, M. Electro-Spinning of Pure Collagen Nano-Fibres-Just an Expensive Way to Make Gelatin? Biomaterials 2008, 29, $2293-2305$. [CrossRef]

86. Nottelet, B.; Pektok, E.; Mandracchia, D.; Tille, J.C.; Walpoth, B.; Gurny, R.; Moller, M. Factorial Design Optimization and in Vivo Feasibility of Poly(Epsilon-Caprolactone)-Micro- and Nanofiber-Based Small Diameter Vascular Grafts. J. Biomed. Mater. Res. A 2009, 89, 865-875. [CrossRef] [PubMed]

87. Edwards, A.; Carson, R.J.; Bowald, S.; Quist, W.C. Development of a Microporous Compliant Small Bore Vascular Graft. J. Biomater. Appl. 1995, 10, 171-187. [CrossRef] [PubMed]

88. Hiob, M.A.; She, S.; Muiznieks, L.D.; Weiss, A.S. Biomaterials and Modifications in the Development of Small-Diameter Vascular Grafts. ACS Biomater. Sci. Eng. 2017, 3, 712-723. [CrossRef]

89. Sarkar, S.; Sales, K.M.; Hamilton, G.; Seifalian, A.M. Addressing Thrombogenicity in Vascular Graft Construction. J. Biomed. Mater. Res. B Appl. Biomater. 2007, 82, 100-108. [CrossRef]

90. Hsu, S.H.; Tseng, H.J.; Wu, M.S. Comparative in Vitro Evaluation of Two Different Preparations of Small Diameter Polyurethane Vascular Grafts. Artif. Organs 2000, 24, 119-128. [CrossRef]

91. Tiwari, A.; Salacinski, H.; Seifalian, A.M.; Hamilton, G. New Prostheses for Use in Bypass Grafts with Special Emphasis on Polyurethanes. Cardiovasc. Surg. 2002, 10, 191-197. [CrossRef]

92. Faturechi, R.; Hashemi, A.; Abolfathi, N.; Solouk, A.; Seifalian, A. Fabrications of Small Diameter Compliance Bypass Conduit Using Electrospinning of Clinical Grade Polyurethane. Vascular 2019, 27, 636-647. [CrossRef] [PubMed]

93. Beck, L.R.; Pope, V.Z.; Flowers, C.E., Jr.; Cowsar, D.R.; Tice, T.R.; Lewis, D.H.; Dunn, R.L.; Moore, A.B.; Gilley, R.M. Poly(DlLactide-Co-Glycolide)/Norethisterone Microcapsules: An Injectable Biodegradable Contraceptive. Biol. Reprod. 1983, 28 , 186-195. [CrossRef]

94. Bettinger, C.J.; Orrick, B.; Misra, A.; Langer, R.; Borenstein, J.T. Microfabrication of Poly (Glycerol-Sebacate) for Contact Guidance Applications. Biomaterials 2006, 27, 2558-2565. [CrossRef]

95. Cutright, D.E.; Perez, B.; Beasley, J.D., 3rd; Larson, W.J.; Posey, W.R. Degradation Rates of Polymers and Copolymers of Polylactic and Polyglycolic Acids. Oral Surg. Oral Med. Oral Pathol. 1974, 37, 142-152. [CrossRef]

96. Marchant, R.E.; Zhao, Q.; Anderson, J.M.; Hiltner, A. Degradation of a Poly(Ether Urethane Urea) Elastomer-Infrared and Xps Studies. Polymer 1987, 28, 2032-2039. [CrossRef]

97. von Recum, H.A.; Cleek, R.L.; Eskin, S.G.; Mikos, A.G. Degradation of Polydispersed Poly(L-Lactic Acid) to Modulate Lactic Acid Release. Biomaterials 1995, 16, 441-447. [CrossRef]

98. Tainio, J.; Paakinaho, K.; Ahola, N.; Hannula, M.; Hyttinen, J.; Kellomaki, M.; Massera, J. In Vitro Degradation of Borosilicate Bioactive Glass and Poly(L-Lactide-Co-Epsilon-Caprolactone) Composite Scaffolds. Materials 2017, 10, 1274. [CrossRef]

99. Awad, N.K.; Niu, H.; Ali, U.; Morsi, Y.S.; Lin, T. Electrospun Fibrous Scaffolds for Small-Diameter Blood Vessels: A Review. Membranes 2018, 8, 15. [CrossRef]

100. Shin'oka, T.; Imai, Y.; Ikada, Y. Transplantation of a Tissue-Engineered Pulmonary Artery. N. Engl. J. Med. 2001, $344,532-533$. [CrossRef]

101. Hibino, N.; McGillicuddy, E.; Matsumura, G.; Ichihara, Y.; Naito, Y.; Breuer, C.; Shinoka, T. Late-Term Results of Tissue-Engineered Vascular Grafts in Humans. J. Thorac. Cardiovasc. Surg. 2010, 139, 431-436. [CrossRef]

102. Shin'oka, T.; Matsumura, G.; Hibino, N.; Naito, Y.; Watanabe, M.; Konuma, T.; Sakamoto, T.; Nagatsu, M.; Kurosawa, H. Midterm Clinical Result of Tissue-Engineered Vascular Autografts Seeded with Autologous Bone Marrow Cells. J. Thorac. Cardiovasc. Surg. 2005, 129, 1330-1338. [CrossRef] [PubMed]

103. Bockeria, L.A.; Svanidze, O.; Kim, A.; Shatalov, K.; Makarenko, V.; Cox, M.; Carrel, T. Total Cavopulmonary Connection with a New Bioabsorbable Vascular Graft: First Clinical Experience. J. Thorac. Cardiovasc. Surg. 2017, 153, 1542-1550. [CrossRef] [PubMed]

104. Guhathakurta, S.; Galla, S.; Ramesh, B.; Venugopal, J.R.; Ramakrishna, S.; Cherian, K.M. Nanofiber-Reinforced Biological Conduit in Cardiac Surgery: Preliminary Report. Asian Cardiovasc. Thorac. Ann. 2011, 19, 207-212. [CrossRef] [PubMed]

105. Burkel, W.E. The Challenge of Small Diameter Vascular Grafts. Med Prog Technol 1988, 14, 165-175. [PubMed]

106. Spanos, H.G. Aspirin Fails to Inhibit Platelet Aggregation in Sheep. Thromb. Res. 1993, 72, 175-182. [CrossRef]

107. Connell, J.M.; Khalapyan, T.; Al-Mondhiry, H.A.; Wilson, R.P.; Rosenberg, G.; Weiss, W.J. Anticoagulation of Juvenile Sheep and Goats with Heparin, Warfarin, and Clopidogrel. Asaio J. 2007, 53, 29-37. [CrossRef]

108. Pfeiffer, D.; Stefanitsch, C.; Wankhammer, K.; Muller, M.; Dreyer, L.; Krolitzki, B.; Zernetsch, H.; Glasmacher, B.; Lindner, C.; Lass, A.; et al. Endothelialization of Electrospun Polycaprolactone (Pcl) Small Caliber Vascular Grafts Spun from Different Polymer Blends. J. Biomed. Mater. Res. A 2014, 102, 4500-4509. [CrossRef] 
109. Woodruff, M.A.; Hutmacher, D.W. The Return of a Forgotten Polymer-Polycaprolactone in the 21st Century. Prog. Polym. Sci. 2010, 35, 1217-1256. [CrossRef]

110. Miller, K.S.; Khosravi, R.; Breuer, C.K.; Humphrey, J.D. A Hypothesis-Driven Parametric Study of Effects of Polymeric Scaffold Properties on Tissue Engineered Neovessel Formation. Acta Biomater. 2015, 11, 283-294. [CrossRef]

111. Malafaya, P.B.; Silva, G.A.; Reis, R.L. Natural-Origin Polymers as Carriers and Scaffolds for Biomolecules and Cell Delivery in Tissue Engineering Applications. Adv. Drug Deliv. Rev. 2007, 59, 207-233. [CrossRef]

112. Nocera, A.D.; Comin, R.; Salvatierra, N.A.; Cid, M.P. Development of 3d Printed Fibrillar Collagen Scaffold for Tissue Engineering. Biomed. Microdevices 2018, 20, 26. [CrossRef]

113. Ramaswamy, A.K.; Vorp, D.A.; Weinbaum, J.S. Functional Vascular Tissue Engineering Inspired by Matricellular Proteins. Front Cardiovasc. Med. 2019, 6, 74. [CrossRef]

114. Wang, Z.; Mithieux, S.M.; Weiss, A.S. Fabrication Techniques for Vascular and Vascularized Tissue Engineering. Adv. Healthc. Mater. 2019, 8, e1900742. [CrossRef] [PubMed]

115. Badylak, S.F. Decellularized Allogeneic and Xenogeneic Tissue as a Bioscaffold for Regenerative Medicine: Factors That Influence the Host Response. Ann. Biomed. Eng. 2014, 42, 1517-1527. [CrossRef] [PubMed]

116. Gilpin, A.; Yang, Y. Decellularization Strategies for Regenerative Medicine: From Processing Techniques to Applications. Biomed. Res. Int. 2017, 2017, 9831534. [CrossRef]

117. Raya-Rivera, A.M.; Esquiliano, D.; Fierro-Pastrana, R.; Lopez-Bayghen, E.; Valencia, P.; Ordorica-Flores, R.; Soker, S.; Yoo, J.J.; Atala, A. Tissue-Engineered Autologous Vaginal Organs in Patients: A Pilot Cohort Study. Lancet 2014, 384, 329-336. [CrossRef]

118. Cebotari, S.; Lichtenberg, A.; Tudorache, I.; Hilfiker, A.; Mertsching, H.; Leyh, R.; Breymann, T.; Kallenbach, K.; Maniuc, L.; Batrinac, A.; et al. Clinical Application of Tissue Engineered Human Heart Valves Using Autologous Progenitor Cells. Circulation 2006, 114, I132-I137. [CrossRef] [PubMed]

119. Dohmen, P.M.; Lembcke, A.; Holinski, S.; Pruss, A.; Konertz, W. Ten Years of Clinical Results with a Tissue-Engineered Pulmonary Valve. Ann. Thorac. Surg. 2011, 92, 1308-1314. [CrossRef]

120. Kneib, C.; von Glehn, C.Q.; Costa, F.D.; Costa, M.T.; Susin, M.F. Evaluation of Humoral Immune Response to Donor Hla after Implantation of Cellularized Versus Decellularized Human Heart Valve Allografts. Tissue Antigens 2012, 80, 165-174. [CrossRef]

121. Kirkton, R.D.; Santiago-Maysonet, M.; Lawson, J.H.; Tente, W.E.; Dahl, S.L.M.; Niklason, L.E.; Prichard, H.L. Bioengineered Human Acellular Vessels Recellularize and Evolve into Living Blood Vessels after Human Implantation. Sci. Transl. Med. 2019, 11. [CrossRef]

122. Skovrind, I.; Harvald, E.B.; Belling, H.J.; Jorgensen, C.D.; Lindholt, J.S.; Andersen, D.C. Concise Review: Patency of SmallDiameter Tissue-Engineered Vascular Grafts: A Meta-Analysis of Preclinical Trials. Stem. Cells Transl. Med. 2019, 8, 671-680. [CrossRef]

123. Nagiah, N.; Johnson, R.; Anderson, R.; Elliott, W.; Tan, W. Highly Compliant Vascular Grafts with Gelatin-Sheathed Coaxially Structured Nanofibers. Langmuir 2015, 31, 12993-13002. [CrossRef] [PubMed]

124. Campbell, J.H.; Efendy, J.L.; Campbell, G.R. Novel Vascular Graft Grown within Recipient's Own Peritoneal Cavity. Circ. Res. 1999, 85, 1173-1178. [CrossRef]

125. Verhagen, H.J.; Heijnen-Snyder, G.J.; Pronk, A.; Vroom, T.M.; van Vroonhoven, T.J.; Eikelboom, B.C.; Sixma, J.J.; de Groot, P.G. Thrombomodulin Activity on Mesothelial Cells: Perspectives for Mesothelial Cells as an Alternative for Endothelial Cells for Cell Seeding on Vascular Grafts. Br. J. Haematol. 1996, 95, 542-549. [CrossRef]

126. Geelhoed, W.J.; Moroni, L.; Rotmans, J.I. Utilizing the Foreign Body Response to Grow Tissue Engineered Blood Vessels in Vivo. J. Cardiovasc. Transl. Res. 2017, 10, 167-179. [CrossRef] [PubMed]

127. Furukoshi, M.; Moriwaki, T.; Nakayama, Y. Development of an in Vivo Tissue-Engineered Vascular Graft with Designed Wall Thickness (Biotube Type C) Based on a Novel Caged Mold. J. Artif. Organs 2016, 19, 54-61. [CrossRef] [PubMed]

128. Zhu, M.; Li, W.; Dong, X.; Yuan, X.; Midgley, A.C.; Chang, H.; Wang, Y.; Wang, H.; Wang, K.; Ma, P.X.; et al. In Vivo Engineered Extracellular Matrix Scaffolds with Instructive Niches for Oriented Tissue Regeneration. Nat. Commun. 2019, 10, 1-14. [CrossRef]

129. Furie, B.; Furie, B.C. Mechanisms of Thrombus Formation. N. Engl. J. Med. 2008, 359, 938-949. [CrossRef]

130. Hu, Z.; Zhang, D.Y.; Lu, S.T.; Li, P.W.; Li, S.D. Chitosan-Based Composite Materials for Prospective Hemostatic Applications. Mar. Drugs 2018, 16, 273. [CrossRef] [PubMed]

131. Adipurnama, I.; Yang, M.C.; Ciach, T.; Butruk-Raszeja, B. Surface Modification and Endothelialization of Polyurethane for Vascular Tissue Engineering Applications: A Review. Biomater. Sci. 2016, 5, 22-37. [CrossRef]

132. Tatterton, M.; Wilshaw, S.P.; Ingham, E.; Homer-Vanniasinkam, S. The Use of Antithrombotic Therapies in Reducing Synthetic Small-Diameter Vascular Graft Thrombosis. Vasc. Endovasc. Surg. 2012, 46, 212-222. [CrossRef]

133. Sanchez, P.F.; Brey, E.M.; Briceno, J.C. Endothelialization Mechanisms in Vascular Grafts. J. Tissue Eng. Regen. Med. 2018, 12, 2164-2178. [CrossRef]

134. Wadey, K.; Lopes, J.; Bendeck, M.; George, S. Role of Smooth Muscle Cells in Coronary Artery Bypass Grafting Failure. Cardiovasc. Res. 2018, 114, 601-610. [CrossRef]

135. Liu, J.; Qin, Y.; Wu, Y.; Sun, Z.; Li, B.; Jing, H.; Zhang, C.; Li, C.; Leng, X.; Wang, Z.; et al. The Surrounding Tissue Contributes to Smooth Muscle Cells' Regeneration and Vascularization of Small Diameter Vascular Grafts. Biomater. Sci. 2019, 7, 914-925. [CrossRef] 
136. Gomes, D.; Louedec, L.; Plissonnier, D.; Dauge, M.C.; Henin, D.; Osborne-Pellegrin, M.; Michel, J.B. Endoluminal Smooth Muscle Cell Seeding Limits Intimal Hyperplasia. J. Vasc. Surg. 2001, 34, 707-715. [CrossRef]

137. Butler, D.L.; Hunter, S.A.; Chokalingam, K.; Cordray, M.J.; Shearn, J.; Juncosa-Melvin, N.; Nirmalanandhan, S.; Jain, A. Using Functional Tissue Engineering and Bioreactors to Mechanically Stimulate Tissue-Engineered Constructs. Tissue Eng. Part A 2009, 15, 741-749. [CrossRef]

138. Kamiya, A.; Bukhari, R.; Togawa, T. Adaptive Regulation of Wall Shear Stress Optimizing Vascular Tree Function. Bull. Math. Biol. 1984, 46, 127-137. [CrossRef]

139. Luciani, N.; Du, V.; Gazeau, F.; Richert, A.; Letourneur, D.; le Visage, C.; Wilhelm, C. Successful Chondrogenesis within Scaffolds, Using Magnetic Stem Cell Confinement and Bioreactor Maturation. Acta Biomater. 2016, 37, 101-110. [CrossRef]

140. Zilla, P.; Bezuidenhout, D.; Human, P. Prosthetic Vascular Grafts: Wrong Models, Wrong Questions and No Healing. Biomaterials 2007, 28, 5009-5027. [CrossRef] [PubMed]

141. Berger, K.; Sauvage, L.R.; Rao, A.M.; Wood, S.J. Healing of Arterial Prostheses in Man: Its Incompleteness. Ann. Surg. 1972, 175, 118-127. [CrossRef] [PubMed]

142. Pennel, T.; Zilla, P.; Bezuidenhout, D. Differentiating Transmural from Transanastomotic Prosthetic Graft Endothelialization through an Isolation Loop-Graft Model. J. Vasc. Surg. 2013, 58, 1053-1061. [CrossRef] [PubMed]

143. Piterina, A.V.; Cloonan, A.J.; Meaney, C.L.; Davis, L.M.; Callanan, A.; Walsh, M.T.; McGloughlin, T.M. Ecm-Based Materials in Cardiovascular Applications: Inherent Healing Potential and Augmentation of Native Regenerative Processes. Int. J. Mol. Sci. 2009, 10, 4375-4417. [CrossRef]

144. Wu, M.H.; Shi, Q.; Wechezak, A.R.; Clowes, A.W.; Gordon, I.L.; Sauvage, L.R. Definitive Proof of Endothelialization of a Dacron Arterial Prosthesis in a Human Being. J. Vasc. Surg. 1995, 21, 862-867. [CrossRef]

145. Zhang, Z.; Briana, S.; Douville, Y.; Zhao, H.; Gilbert, N. Transmural Communication at a Subcellular Level May Play a Critical Role in the Fallout Based-Endothelialization of Dacron Vascular Prostheses in Canine. J. Biomed. Mater. Res. A 2007, 81, 877-887. [CrossRef]

146. Melchiorri, A.J.; Hibino, N.; Fisher, J.P. Strategies and Techniques to Enhance the in Situ Endothelialization of Small-Diameter Biodegradable Polymeric Vascular Grafts. Tissue Eng. Part B Rev. 2013, 19, 292-307. [CrossRef] [PubMed]

147. Tassiopoulos, A.K.; Greisler, H.P. Angiogenic Mechanisms of Endothelialization of Cardiovascular Implants: A Review of Recent Investigative Strategies. J. Biomater. Sci. Polym. Ed. 2000, 11, 1275-1284. [CrossRef]

148. Talacua, H.; Smits, A.I.; Muylaert, D.E.; van Rijswijk, J.W.; Vink, A.; Verhaar, M.C.; Driessen-Mol, A.; van Herwerden, L.A.; Bouten, C.V.; Kluin, J.; et al. In Situ Tissue Engineering of Functional Small-Diameter Blood Vessels by Host Circulating Cells Only. Tissue Eng. Part A 2015, 21, 2583-2594. [CrossRef] [PubMed]

149. Ren, X.; Feng, Y.; Guo, J.; Wang, H.; Li, Q.; Yang, J.; Hao, X.; Lv, J.; Ma, N.; Li, W. Surface Modification and Endothelialization of Biomaterials as Potential Scaffolds for Vascular Tissue Engineering Applications. Chem. Soc. Rev. 2015, 44, 5680-5742. [CrossRef]

150. Balistreri, C.R.; Buffa, S.; Pisano, C.; Lio, D.; Ruvolo, G.; Mazzesi, G. Are Endothelial Progenitor Cells the Real Solution for Cardiovascular Diseases? Focus on Controversies and Perspectives. Biomed. Res. Int. 2014, 2015. [CrossRef] [PubMed]

151. Qiu, J.; Hirschi, K.K. Endothelial Cell Development and Its Application to Regenerative Medicine. Circ. Res. 2019, 125, 489-501. [CrossRef]

152. Basile, P.D.; Yoder, M.C. Circulating and Tissue Resident Endothelial Progenitor Cells. J. Cell. Physiol. 2014, 229, 10-16. [CrossRef] [PubMed]

153. Scharn, D.M.; Daamen, W.F.; van Kuppevelt, T.H.; van der Vliet, J.A. Biological Mechanisms Influencing Prosthetic Bypass Graft Patency: Possible Targets for Modern Graft Design. Eur. J. Vasc. Endovasc. Surg. 2012, 43, 66-72. [CrossRef]

154. Jiang, B.; Suen, R.; Wertheim, J.A.; Ameer, G.A. Targeting Heparin to Collagen within Extracellular Matrix Significantly Reduces Thrombogenicity and Improves Endothelialization of Decellularized Tissues. Biomacromolecules 2016, 17, 940-948. [CrossRef]

155. Zhu, Y.; Gao, C.; Shen, J. Surface Modification of Polycaprolactone with Poly(Methacrylic Acid) and Gelatin Covalent Immobilization for Promoting Its Cytocompatibility. Biomaterials 2002, 23, 4889-4895. [CrossRef]

156. Radke, D.; Jia, W.; Sharma, D.; Fena, K.; Wang, G.; Goldman, J.; Zhao, F. Tissue Engineering at the Blood-Contacting Surface: A Review of Challenges and Strategies in Vascular Graft Development. Adv. Healthc. Mater. 2018, 7, e1701461. [CrossRef]

157. Collins, M.J.; Li, X.; Lv, W.; Yang, C.; Protack, C.D.; Muto, A.; Jadlowiec, C.C.; Shu, C.; Dardik, A. Therapeutic Strategies to Combat Neointimal Hyperplasia in Vascular Grafts. Expert Rev. Cardiovasc. Ther. 2012, 10, 635-647. [CrossRef]

158. Fukuda, D.; Aikawa, M. Intimal Smooth Muscle Cells: The Context-Dependent Origin. Circulation 2010, 122, 2005-2008. [CrossRef] [PubMed]

159. Yokote, K.; Take, A.; Nakaseko, C.; Kobayashi, K.; Fujimoto, M.; Kawamura, H.; Maezawa, Y.; Nishimura, M.; Mori, S.; Saito, Y. Bone Marrow-Derived Vascular Cells in Response to Injury. J. Atheroscler. Thromb. 2003, 10, 205-210. [CrossRef]

160. Kijani, S.; Vazquez, A.M.; Levin, M.; Boren, J.; Fogelstrand, P. Intimal Hyperplasia Induced by Vascular Intervention Causes Lipoprotein Retention and Accelerated Atherosclerosis. Physiol. Rep. 2017, 5. [CrossRef] [PubMed]

161. Schwartz, S.M.; deBlois, D.; O’Brien, E.R. The Intima. Soil for Atherosclerosis and Restenosis. Circ. Res. 1995, 77, 445-465. [CrossRef] [PubMed]

162. Anderson, D.E.J.; Pohan, G.; Raman, J.; Konecny, F.; Yim, E.K.F.; Hinds, M.T. Improving Surgical Methods for Studying Vascular Grafts in Animal Models. Tissue Eng. Part C Methods 2018, 24, 457-464. [CrossRef]

163. Matthiasson, S.E.; Bergqvist, D.; Lundell, A.; Lindblad, B. Effect of Dexdtran and Enoxaparin on Early Eptfe Graft Thrombogenicity in Sheep. Eur. J. Vasc. Endovasc. Surg. 1995, 9, 284-292. [CrossRef] 